Sección Básica / Basic

Artículos de investigación/ Research paper

\title{
Abejas como prestadoras de servicios ecosistémicos culturales: caso Jardín Botánico de Bogotá, Colombia
}

\author{
Bees as providers of cultural ecosystem services: case of the Bogotá \\ Botanical Garden, Colombia \\ (iD ÁNGELA RODRÍGUEZ-C. ${ }^{12 *}$; iD VIVIANA GARCÍA-M.3; \\ (iD SUSANA CURREA-M. ${ }^{2}$
}

${ }^{1}$ Universidad Nacional de Colombia, Bogotá, Colombia.atrodriguezc@unal.edu.co, sucurreamo@unal.edu.co² Jardín Botánico José Celestino Mutis, Bogotá, Colombia. ${ }^{3}$ Universidad INCCA de Colombia. Bogotá, Colombia, vivigar26@gmail.com

\begin{abstract}
*Autor para correspondencia
Ángela Rodríguez, Bióloga M.Sc, Universidad Nacional de Colombia, Carrera 30 \# 45 - 03, Bogotá, Colombia, atrodriguezc@unal.edu.co.

\section{Citación sugerida}

RODRÍGUEZ-C., A.; GARCÍA-M., V.; CURREA-M., S. 2021. Abejas como prestadoras de servicios ecosistémicos culturales: caso Jardín Botánico de Bogotá, Colombia. Revista Colombiana de Entomología 47 (2): e10560. https:// doi.org/10.25100/socolen.v47i2.10560

Recibido: 19-Ago-2020

Aceptado: 09-Abr-2021

Publicado: 20-sep-2021

Revista Colombiana de Entomología ISSN (Print): 0120-0488

ISSN (On Line): 2665-4385

https://revistacolombianaentomologia.univalle.edu.co

Open access

(c) (i) (2) (2) BY-NC-SA 4.0
\end{abstract}

Publishers: Sociedad Colombiana de Entomología SOCOLEN (Bogotá, D. C., Colombia) https://www.socolen.org.co

Universidad del Valle (Cali, Colombia)

https://www.univalle.edu.co

(C) 2021 Sociedad Colombiana de Entomología SOCOLEN y Universidad del Valle - Univalle
Resumen: El propósito de este estudio fue evaluar el potencial de los domicilios artificiales para abejas solitarias y de esta manera explorar servicios ecosistémicos adicionales a la polinización. Se instalaron módulos artificiales en tres zonas del Jardín Botánico de Bogotá previamente seleccionadas. Se realizaron monitoreos semanales de junio (2017) a agosto (2019), un monitoreo exhaustivo cada seis meses y el análisis palinológico de 48 muestras recolectadas de los módulos. Sólo el módulo de páramo y bosque altoandino $(33,3 \%)$ presentó colonización por abejas (Megachile amparo, Megachile sp., Colletes sp. y Chilicola brooksi). Los materiales más usados como nidos fueron los bloques de madera perforada y los tubos de papel. La tasa de emergencia de nidos incubados fue mayor en Colletes sp. y no se observó un orden en la emergencia de hembras y machos, mientras que en $M$. amparo la emergencia de machos fue mayor. Las hembras de Colletes sp. presentaron la mayor colonización de nidos en el estudio. El análisis de polen de los nidos permitió evidenciar que Colletes sp. tiene tendencia a ser oligoléctica, mientras que $M$. amparo y $C$. brooksi son polilécticas. El bajo número de especies que nidificó en estos módulos puede indicar un reducido potencial como estrategia de conservación, sin embargo, fueron herramientas útiles de investigación para conocer hábitos de especies con escasa o nula información. Los módulos permitieron tener interacciones experienciales, educativas, científicas y estéticas como formas de servicios ecosistémicos culturales, así como formular recomendaciones para considerar la pertinencia de su implementación.

Palabras clave: abejas urbanas, Colletes, educación ambiental, Megachile amparo, nidos trampa.

Abstract: The purpose of this study was to evaluate the potential of artificial domiciles for solitary bees and thereby explore ecosystem services besides pollination. Artificial modules were installed in three previously selected areas of the Botanical Garden of Bogotá. Weekly monitoring was carried out from June (2017) to August (2019), an exhaustive monitoring every six months and the palynological analysis of 48 samples collected from the modules. Only the Páramo and high Andean Forest module (33.3 \%) was colonised by bees (Megachile amparo, Megachile sp., Colletes sp. and Chilicola brooksi). The materials most used as nests were perforated wooden blocks and paper tubes. The emergency rate of incubated nests was higher in Colletes sp., and no order was observed in the emergence of females and males, while in M. amparo the emergence of males was greater. The females of Colletes sp. presented the highest colonization of nests in the study. Pollen analysis of the nests showed that Colletes sp. has a tendency to be oligolectic, while M. amparo and C. brooksi are polylectic. The low number of species that nested in these modules may be indicative of the low potential as a conservation strategy; however, they were useful research tools to understand the habits of species with little or no information. The modules made it possible to have experiential, educational, scientific and aesthetic interactions as forms of cultural ecosystem services, as well as to formulate recommendations to consider the relevance of their implementation.

Keywords: Colletes, Megachile amparo, environmental education, trap nests, urban bees.

\section{Introducción}

El concepto de Servicios Ecosistémicos (SE), sin ser el único, es uno de los más extendidos para valorar los beneficios de la naturaleza. Es una propuesta del Millennium Ecosystem Assessment, en la cual se toma como base la cobertura de las necesidades humanas y se definen los SE como los beneficios tangibles e intangibles que obtienen las personas a partir del funcionamiento natural de los ecosistemas (Reid 
et al. 2005). Estos autores definen cuatro categorías de SE: i) servicios de provisión de productos de uso humano directo, ii) servicios de regulación de procesos de los ecosistemas que ofrecen bienestar y protección, iii) servicios culturales o beneficios inmateriales y iv) servicios de base necesarios para proveer todos los otros SE (Reid et al. 2005).

Para el caso de los SE prestados por insectos se encontró que estos aportan en tres categorías principales: i) de provisión, ii) de regulación y iii) culturales, además resaltan la falta de reconocimiento del aporte de los insectos como proveedores de SE de provisión y culturales (Ameixa et al. 2018). Si bien, para el caso de las abejas el SE más destacado es la polinización, el cual hace parte de los SE de base (Klein et al. 2006; Bonilla 2017; Ramos et al. 2020), se deben reconocer los aportes en términos de investigación, educación, patrimonio, entretenimiento y estética, los cuales son escasamente estudiados y valorados.

Está demostrado que los espacios verdes urbanos por su heterogeneidad y microhábitats actúan como ecosistemas de refugio y son importantes en la conservación de una gran variedad de organismos, lo que los hace útiles para realizar estrategias ante la crisis de polinizadores, por ello existe un creciente interés de investigar y ayudar a los polinizadores en entornos urbanos (Mader et al. 2010; Winfree et al. 2011; Khew y Yokohari 2017; Siemaszko y Zych 2017; Mason y Arathi 2019). Muchos de estos proyectos están enfocados en estudiar abejas usando diversos métodos, como los nidos trampa y la adecuación de espacios como jardines con el fin de proveer recursos florales y atraer organismos benéficos (Wratten et al. 2012; MacIvor 2016; Hall et al. 2017; Gardiner y Fargeaud 2018; Langellotto et al. 2018; Turo y Gardiner 2019). En Colombia los estudios de abejas urbanas se han enfocado principalmente en las abejas sociales Meliponini y en sus hábitos de nidificación (Nates-Parra et al. 2006b; Vélez-Ruiz et al. 2013; Martínez et al. 2017). Respecto a Bogotá se han descrito nuevas especies urbanas y se ha profundizado sobre los hábitos de nidificación y forrajeo de 21 de las 40 especies reportadas para la ciudad (González 2006; Nates-Parra et al. 2006a; Tellez-Farfán y Posada-Flórez 2013; Pinilla-Gallego et al. 2016; Rodríguez-C. et al. 2019). Sin embargo, aún se requiere continuar con estudios de varias especies de abejas solitarias para conocer más sobre su biología y diseñar estrategias de conservación.

Un factor crítico para la presencia de abejas es la oferta de sustratos para nidificar, la cual está condicionada por la variedad de comportamientos y preferencias de nidificación como el suelo, tallos huecos o cavidades preexistentes (Tscharntke et al. 1998; Michener 2000; Cane 2015). Incluso, está reportado que algunos grupos tienen una alta adaptabilidad a ambientes transformados, y que han empezado a incluir en la arquitectura de sus nidos materiales de origen antrópico (Kamel et al. 2007; Fortel et al. 2016; Allasino et al. 2019). En respuesta a esta necesidad, en muchos trabajos académicos y ciudadanos, se ha probado gran variedad de diseños de domicilios artificiales para abejas, denominados popularmente 'hoteles' (Mader et al. 2010; Halcroft 2012; Bauer et al. 2015; MacIvor y Packer 2015; Carlton 2017; MacIvor 2017; Nates-Parra 2017b; Staab et al. 2018; Tucker et al. 2019).

A nivel de investigación los nidos trampa han sido útiles para el estudio del comportamiento y la descripción de los nidos de abejas solitarias y otros organismos asociados (Gathmann et al. 1994; Wcislo 1996; MacIvor y Salehi 2014; Kumar et al. 2015). Sin embargo, su aporte a la conserva- ción es motivo de controversia (MacIvor y Packer 2015), a pesar de que hoy en el comercio es frecuente encontrar hoteles prefabricados que se promocionan con este propósito (Carlton 2017). Sin evidencias, resulta difícil afirmar que la instalación de estos objetos es una estrategia exitosa para conservar poblaciones de abejas silvestres, en especial cuando existe una gran cantidad de especies que tienen otros comportamientos de nidificación y por lo cual nunca llegarán a usar estas estructuras (Cane 2015). De igual forma, algunos autores sugieren que para la conservación de polinizadores es importante tener en cuenta variables sociales, en especial aquellas que pueden tener un impacto en los manejos y estrategias aplicadas (Siemaszko y Zych 2017; Staab et al. 2018).

Por esto es fundamental que dichas iniciativas cuenten con bases científicas y que, además, contemplen para su aplicación el impacto que tendrían tanto para las personas como para las poblaciones de abejas (disminución de diversidad genética, aumento de parasitismo y otras afecciones). Los jardines botánicos pueden funcionar como espacios propicios donde el conocimiento científico y los ciudadanos interesados confluyan. Ya es posible encontrar iniciativas similares e incluso hoteles de abejas en zonas verdes de ciudades y, en concreto, en jardines botánicos (Mazzeo y Torretta 2015; RJB 2017; BGM 2019; Smith y Miller 2020).

El Jardín Botánico de Bogotá (JBB) desde 2014 mantiene proyectos de investigación enfocados en conocer la fauna Apoidea presente en sus instalaciones. Como resultado de estas investigaciones se realizó un inventario inicial de 16 especies de abejas con representantes de las cinco familias de Apoidea reportadas para Colombia (Rodríguez-C. et al. 2019). A raíz de estos hallazgos, se planteó usar domicilios artificiales que propiciarán la presencia de abejas en los predios del Jardín, y así evaluar su potencial para estrategias de investigación, conservación y educación. En este estudio se presentan los resultados de datos tomados en el Jardín Botánico de Bogotá y se proveen lineamientos básicos sobre la implementación de domicilios artificiales para abejas solitarias como agentes de servicios ecosistémicos culturales.

\section{Materiales y métodos}

Localidad. El Jardín Botánico José Celestino Mutis se encuentra al occidente de la zona urbana de Bogotá D.C., capital de Colombia $\left(04^{\circ} 40^{\prime} 06^{\prime \prime} \mathrm{N}, 74^{\circ} 06^{\prime} 00^{\prime \prime} \mathrm{O}, 2.577 \mathrm{~m}\right.$ de altitud). Contiene colecciones vivas de plantas organizadas en secciones que representan diferentes ecosistemas de Colombia, cuenta con colecciones temáticas de plantas exóticas, de uso ornamental, medicinal, agroecológico, colecciones especializadas en grupos de interés para conservación, entre otras. En estas colecciones también habita una gran variedad de especies de fauna como aves, reptiles, anfibios e insectos.

Instalación de módulos artificiales. Partiendo del inventario de abejas de JBB realizado en 2014 y 2015 (Rodríguez-C. et al. 2019), en 2017 se seleccionaron tres zonas para la instalación de módulos de domicilios artificiales para abejas solitarias: El herbal de la salud (colección de plantas medicinales), zona de páramo y bosque alto andino (colección de vegetación representativa de los paisajes circundantes de Bogotá D.C.) y colección especializada para la conservación de labiadas (colección de especies de la familia Lamiaceae). En cada sitio se instaló un módulo como se muestra en la Figura 1. 


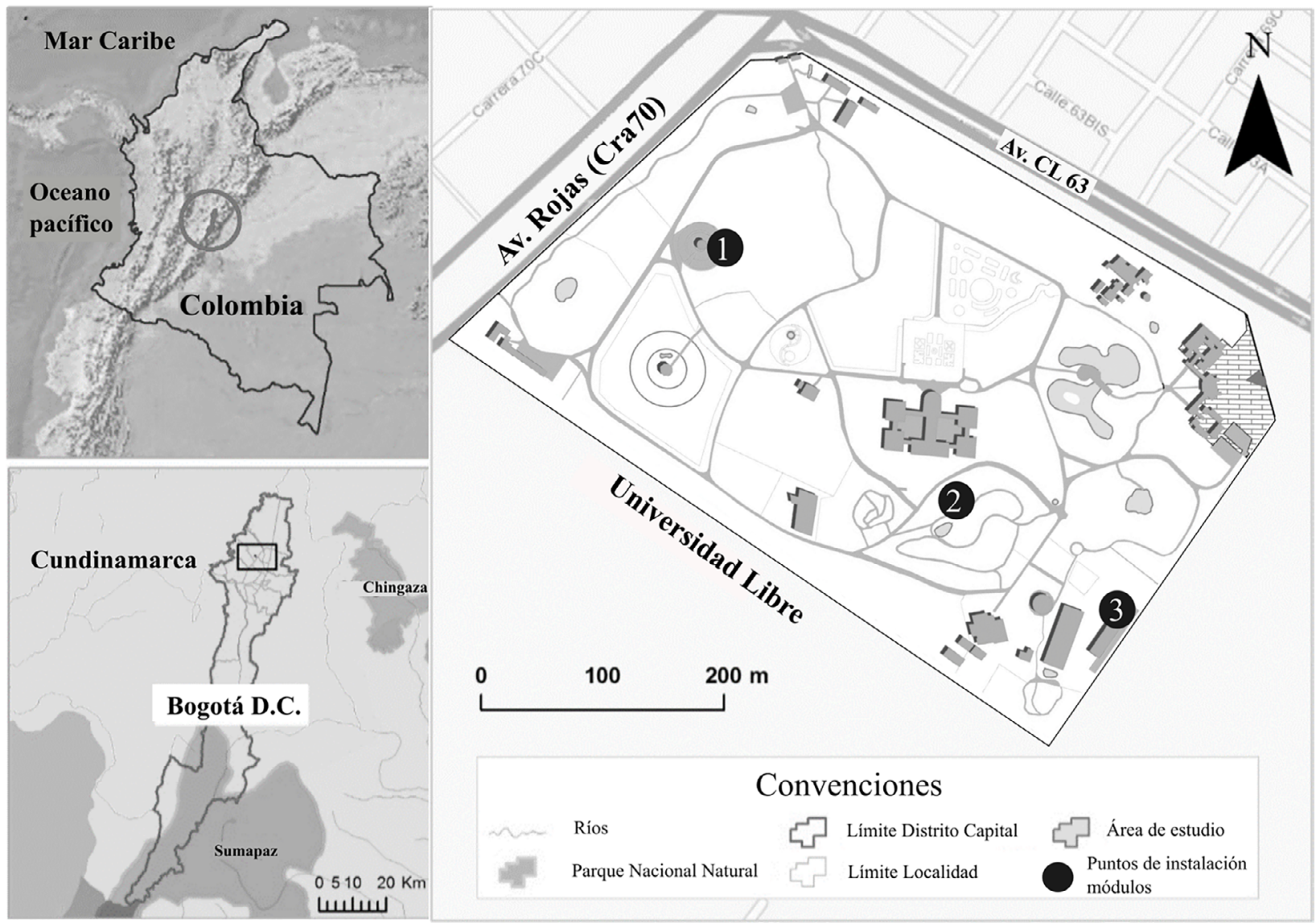

Figura 1. Ubicación del área de estudio y de los módulos instalados. Los puntos del Jardín Botánico donde fueron instalados los módulos se indican con círculos negros: 1 . Herbal de la salud; 2 . Zona de páramo y bosque alto andino; 3 . Colección especializada para la conservación de labiadas.

El diseño de los módulos se basó en las propuestas de diferentes trabajos (Dean 2010; Bauer et al. 2015; MacIvor 2017). Se usó un modelo hexagonal dividido en triángulos de igual tamaño, en los que se dispusieron diferentes tipos de materiales de prueba (Fig. 2). El marco fue elaborado con madera de Solera del género Cordia L. (Boraginaceae) no inmunizada y fue sellado en el primer año del experimento con cera de Apis mellifera (L., 1758) (Hymenoptera: Apidae), después se aplicó aceite de linaza, material comúnmente usado y recomendado para inmunizar y proteger colmenas (Silva-Garnica et al. 2006), con el fin de disminuir la probabilidad de deterioro por humedad, el diseño incluyó un techo de acrílico transparente y se ubicó $1,5 \mathrm{~m}$ separado del suelo por un tubo metálico enterrado en el piso. Los módulos se ubicaron en zonas con buena exposición al sol y rodeados de plantas con flor.

Algunos de los materiales probados se tomaron de plantas donde se había observado o reportado nidificación por abejas y se obtuvieron de las podas regulares que se realizan en el Jardín Botánico, todos con un largo máximo de $15 \mathrm{~cm}$ y sellados por el extremo posterior con cera de A. mellifera o con el mismo material. Posteriormente, se incorporaron tallos de Rubus spp. (Rosaceae) de 4 a $10 \mathrm{~mm}$ de diámetro, papel negro de $10 \mathrm{~mm}$ de diámetro enrollado, pitillos de papel de $6 \mathrm{~mm}$ de diámetro.

Monitoreo y mantenimiento. Desde junio de 2017 hasta agosto de 2019, se llevaron a cabo monitoreos semanales, conteo de los nidos ocupados, registro de eventos inusuales (sustracción de material, ocupantes no abejas) además de labores de mantenimiento, limpieza, reemplazo de los materiales

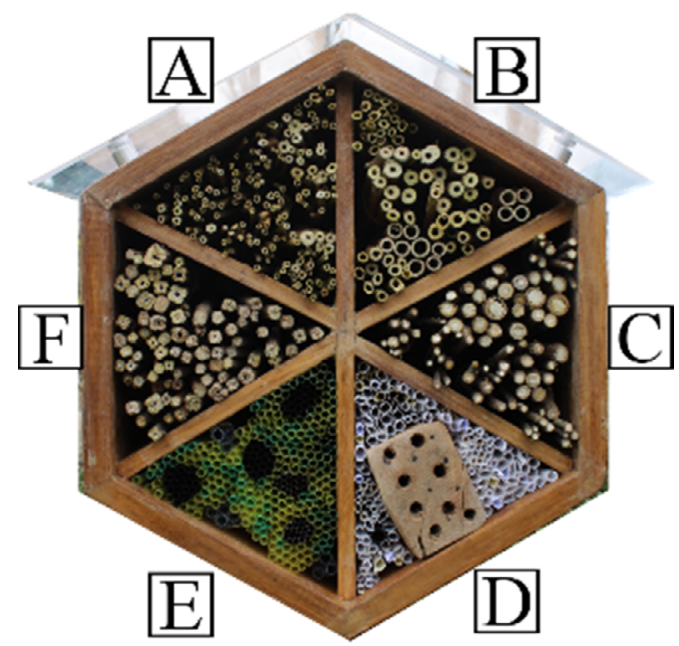

Figura 2. Distribución de tratamientos en el módulo de domicilios, A. Tallos de Monnina sp. (Polygalaceae), diámetro interior $<4 \mathrm{~mm}$, grosor de pared $<3 \mathrm{~mm}$ (190 unidades). B. Tallos de Monnina sp. (Polygalaceae), diámetro interior $>10 \mathrm{~mm}$, grosor de pared 3-8 $\mathrm{mm}$ (96 unidades); cañas de diámetro 5-13 mm (12 unidades). C. Tallos Baccharis sp. (Asteraceae) y Montanoa sp. (Asteraceae) con médula blanda: $>20 \mathrm{~mm}$ de diámetro total (40 unidades); $5-10 \mathrm{~mm}$ de diámetro (50 unidades). D. Bloque de madera de ceiba (nombre comercial para varias especies de la familia Malvaceae) sin inmunizar con perforaciones de 8 y $10 \mathrm{~mm}$; tubos de papel bond $<5 \mathrm{~mm}$ de diámetro (50 unidades); $10 \mathrm{~mm}$ de diámetro (90 unidades). E. Pitillos plásticos $>6 \mathrm{~mm}$ de diámetro (250 unidades); pitillos plásticos $<5 \mathrm{~mm}$ de diámetro (60 unidades); manguera transparente de $8 \mathrm{~mm}$ de diámetro interno (10 unidades). F. Tallos de Salvia spp. (Lamiaceae) $<3 \mathrm{~mm}$ diámetro interior ( 85 unidades). 
sustraídos o deteriorados, observaciones del aspecto externo del módulo y reportes de las prácticas realizadas en su cercanía.

Cada seis meses se realizó un monitoreo exhaustivo, donde los módulos se retiraron y limpiaron. Los tubos se contabilizaron por tipo de material y se reportaron aquellos ocupados por abejas. Algunos de los tubos colonizados se extrajeron para ser observados, medidos y fotografiados en el laboratorio anexo al herbario del Jardín Botánico de Bogotá JBB, allí fueron dispuestos en tubos de vidrio cubiertos con cartulina negra para protegerlos de la luz, con una tapa de tul que evitaba la salida de las abejas y permitía la ventilación. Con el fin de verificar la emergencia de adultos se incubaron a temperaturas entre los $13-19^{\circ} \mathrm{C}$ y $50-75 \%$ de humedad.

Una vez se presentaron los eventos de colonización por abejas, se tomaron muestras de adultos que emergieron de los nidos para su identificación. Se sacrificaron los individuos necesarios para verificar la identidad de las especies y una hembra y un macho de los nidos incubados. Para la identificación se utilizaron claves taxonómicas y guías (Michener et al. 1994; González et al. 2005; González 2006; Smith-Pardo y Vélez 2008), así como la consulta a especialistas. El material entomológico fue montado, etiquetado y depositado en la colección entomológica especializada en abejas LABUN de la Universidad Nacional de Colombia.

Análisis palinológico. Se tomaron 48 muestras de polen provenientes del material contenido en las celdas de los nidos donde emergieron adultos in situ en el módulo y muestras de polen de las celdas de los nidos que se extrajeron e incubaron en el laboratorio. Las muestras se guardaron en viales estériles de $1,5 \mathrm{ml}$ y el material se diluyó en alcohol al $70 \%$. Con ayuda de una varilla de vidrio o pinzas metálicas esterilizadas, el material se maceró contra las paredes del frasco y posteriormente se filtró con una malla metálica. Las muestras que presentaban poco polen se montaron directamente con ayuda de capilares de vidrio en placas con gelatina glicerina coloreada con fucsina.

Para nueve muestras de Megachile spp. en las que la cantidad de polen fue suficiente se llevó a cabo el proceso de acetólisis de Erdtman con las modificaciones propuestas por Fonnegra (1989) en las instalaciones del laboratorio de melisopalinología asociado al laboratorio de investigación en abejas de la Universidad Nacional (LABUN). Se contabilizaron los tipos polínicos encontrados hasta superar los 300 granos por muestra (Nates-Parra et al. 2013). Para la identificación, los tipos polínicos se fotografiaron en 100x con ayuda del ocular-cámara digital AM4023X Dino-Eye USB, y se compararon con muestras de referencia de polen de anteras de las plantas presentes alrededor de los módulos. Para diferenciarlos se tomó en cuenta el ámbito polar, la relación diámetro polar y ecuatorial y la ornamentación de la exina. Se recolectaron en total 37 muestras de anteras de plantas para construir la colección de referencia, estas fueron diluidas en alcohol al $70 \%$, maceradas, filtradas, y luego montadas en placas de microscopio directamente. Adicionalmente, se consultaron catálogos de polen como los de Roubik y Moreno (1991), Giraldo et al. (2011) y Yuca-Rivas (2018).

Divulgación y experiencias de educación. Por el carácter público de la institución en la que se desarrolló el proyecto se fueron implementando estrategias para aumentar paulatinamente el interés del público y disminuir posibles afecta- ciones. Inicialmente los módulos sólo contaban con un aviso de advertencia. Posteriormente, se abrieron espacios con el fin de incluir a la comunidad y a los trabajadores del Jardín, se socializó la investigación en escenarios académicos y espacios internos de la institución y en 2018 se inició la atención de consultas ciudadanas, solicitudes de visita con guía y entrevistas para medios de comunicación sobre los temas de abejas, polinización y hoteles para abejas que surgieron a partir de la divulgación del proyecto. El inventario de estas interacciones se obtuvo mediante consultas a los registros del Sistema Distrital de Quejas y Soluciones del Jardín Botánico y el Registro de visitantes atendidos del Herbario JBB.

\section{Resultados}

Preferencia en los materiales de los nidos trampa. De los tres módulos instalados, sólo el ubicado en la zona de páramo y bosque alto andino presentó colonización por abejas tres meses después de su instalación, por esta razón no se pudieron realizar comparaciones estadísticas, sin embargo, se presentan los hallazgos sobre la biología de las especies que se establecieron (Figura 3). Después de la tercera revisión exhaustiva, se decidió retirar los otros dos módulos no exitosos, y continuar con el monitoreo de los materiales del módulo de la zona de páramo y bosque alto andino. Se observó que el material más usado fue el de los tubos de papel bond blanco, seguido por el bloque de madera perforado y los tallos de Monnina sp. de diámetro mayor a $4 \mathrm{~mm}$.

Una especie del género Colletes (Latreille, 1802) (Hymenoptera: Colletidae), fue la que construyó más nidos en el periodo reportado con 296 nidos a agosto de 2019, mostró preferencia por los tubos de papel bond blanco y bloque de madera perforado, aunque usó una mayor variedad de materiales.

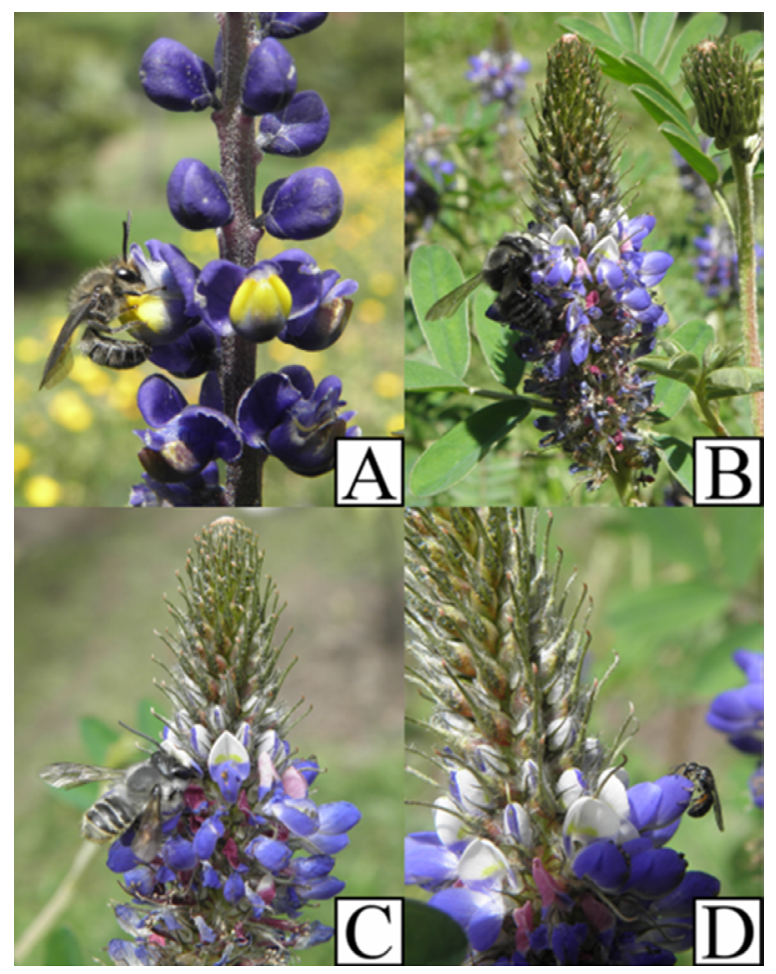

Figura 3. Especies de abejas forrajeando en la zona de páramo y bosque alto andino. A. Colletes sp. en Monnina sp. B. M. amparo en Dalea coerulea (L.f.) Schinz \& Thell (Fabaceae). C. Megachile sp. en D. coerulea. D. C. brooksi en D. coerulea. 
En el caso de Megachile amparo (González, 2006) (Hymenoptera: Megachilidae), mostró una preferencia por el papel bond negro y el bloque de madera perforado. El único evento de nidificación por parte de Chilicola brooksi (Michener, 2002) (Hymenoptera: Colletidae) se presentó en tallos de Monnina sp. con diámetro inferior a $4 \mathrm{~mm}$ y en un pequeño periodo de tiempo se presentó otra especie del género Megachile (Latreille, 1802) (Hymenoptera: Megachilidae), la cual colonizó pocos nidos en bloque de madera. En la Figura 3 se presentan las especies de abejas forrajeando en una zona cerca de los módulos y en la Figura 4 se presentan los resultados para los materiales que fueron colonizados.

El periodo de seguimiento del módulo de domicilios artificiales para abejas tuvo tres fases, una primera de agosto de 2017 a enero de 2018 donde se observó colonización de las cuatro especies reportadas sin aumento significativo del número de nidos. Después de enero de 2018 continuó una segunda fase donde aumentó el número de nidos tanto de $M$. amparo como de Colletes sp. hasta abril de 2018 cuando Colletes sp. comienza a ser dominante como especie colonizadora. En este punto los materiales preferidos ya estaban ocupados con nidos activos, por lo tanto, la tasa de colonización no aumentó y se evidencia disminución a partir de mayo hasta junio de 2018 (Fig. 5). Por lo anterior, se realizó una revisión exhaustiva en junio 2018 en la que se dejaron únicamente los materiales 'exitosos' con signos de colonización y se retiraron aquellos que nunca fueron ocupados, también se seleccionaron nidos para su incubación y análisis. En julio 2018 inició la tercera fase de la investigación en la que el número de nidos de Colletes sp. aumentó y se volvió dominante, tanto que cerca del $100 \%$ del aumento de nidos correspondió a este género.

Arquitectura de nidos. Se realizó el seguimiento en laboratorio y observación de la arquitectura interna de nidos de Colletes sp. y M. amparo. Se extrajeron un total de 56 nidos, (uno de C. brooksi, 17 de Megachile amparo y 37 de
Colletes sp, y un nido con celdas tanto de M. amparo como de Colletes sp.). La tasa de emergencia fue mayor para $\mathrm{Co}$ lletes sp. con emergencias en 29 nidos $(78,37 \%$ de los nidos extraídos), mientras que en $M$. amparo sólo seis nidos tuvieron emergencias $(35,29 \%$ de los nidos extraídos). El tiempo de emergencia del único nido de $C$. brooksi en seguimiento fue de 81 días. El tiempo promedio de emergencia se contó desde el día que las hembras cerraban el nido hasta cuando se veía abierto por primera vez. Los tiempos promedios de emergencia fueron $114 \pm 28,5$ días para Colletes sp. y $115 \pm$ 24,5 días para $M$. amparo.

La proporción hembras/machos que emergieron de los nidos también fue diferente entre nidos, para Colletes sp. el promedio de emergencias por nido fue de 4,5 $\pm 1,8$ abejas, $y$ la proporción de sexos mostró un leve sesgo que tiende hacia una mayor cantidad de machos que de hembras $(1,3: 1)$. En $M$. amparo el promedio de emergencias fue de 2,6 $\pm 1,6$ abejas, siendo machos la mayoría de las emergencias observadas (diez machos y una hembra de cinco nidos totales). Para ninguna de las dos especies se observó un orden claro en la emergencia de machos y hembras; se observaron nidos donde nacían primero hembras y luego machos o viceversa, e incluso nidos donde nacieron intercalados.

Con relación a la arquitectura de los nidos, tanto Colletes sp., como M. amparo construyeron celdas organizadas linealmente sin hacer perforaciones o ramificaciones. Para el caso de Colletes sp., el número de celdas osciló entre dos y seis, cada celda con un único huevo y una provisión húmeda de polen, delimitadas individualmente por bolsitas de un material semejante al celofán (secreción de la glándula de Dufour), este mismo material fue usado para barnizar las paredes interiores de la cavidad y para elaborar el tapón preliminar y el tapón de cierre, los nidos presentaron espacio vacío antes de la primera celda, algunas veces se observaron celdas vestibulares (antes del tapón de cierre) y no se evidenciaron celdas vacías intercalares (entre celdas ocupadas) (Fig. 6).

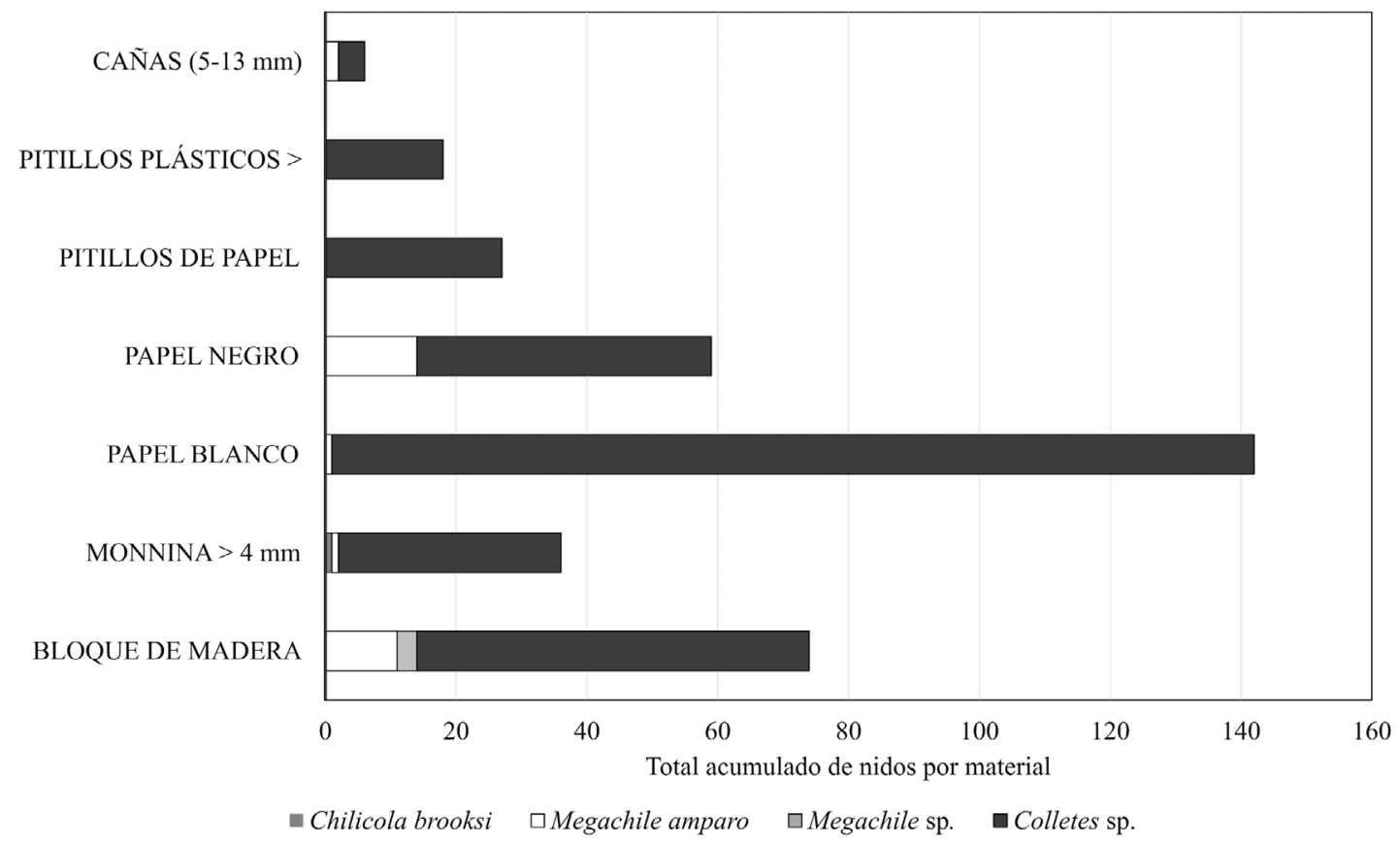

Figura 4. Número acumulado total de nidos por tipo de material. Se presentan el acumulado de nidos construidos en esos materiales y no la proporción respecto a la cantidad de material por tratamiento, debido a que esta cambió con el tiempo y fue afectado por los eventos de vandalismo. 


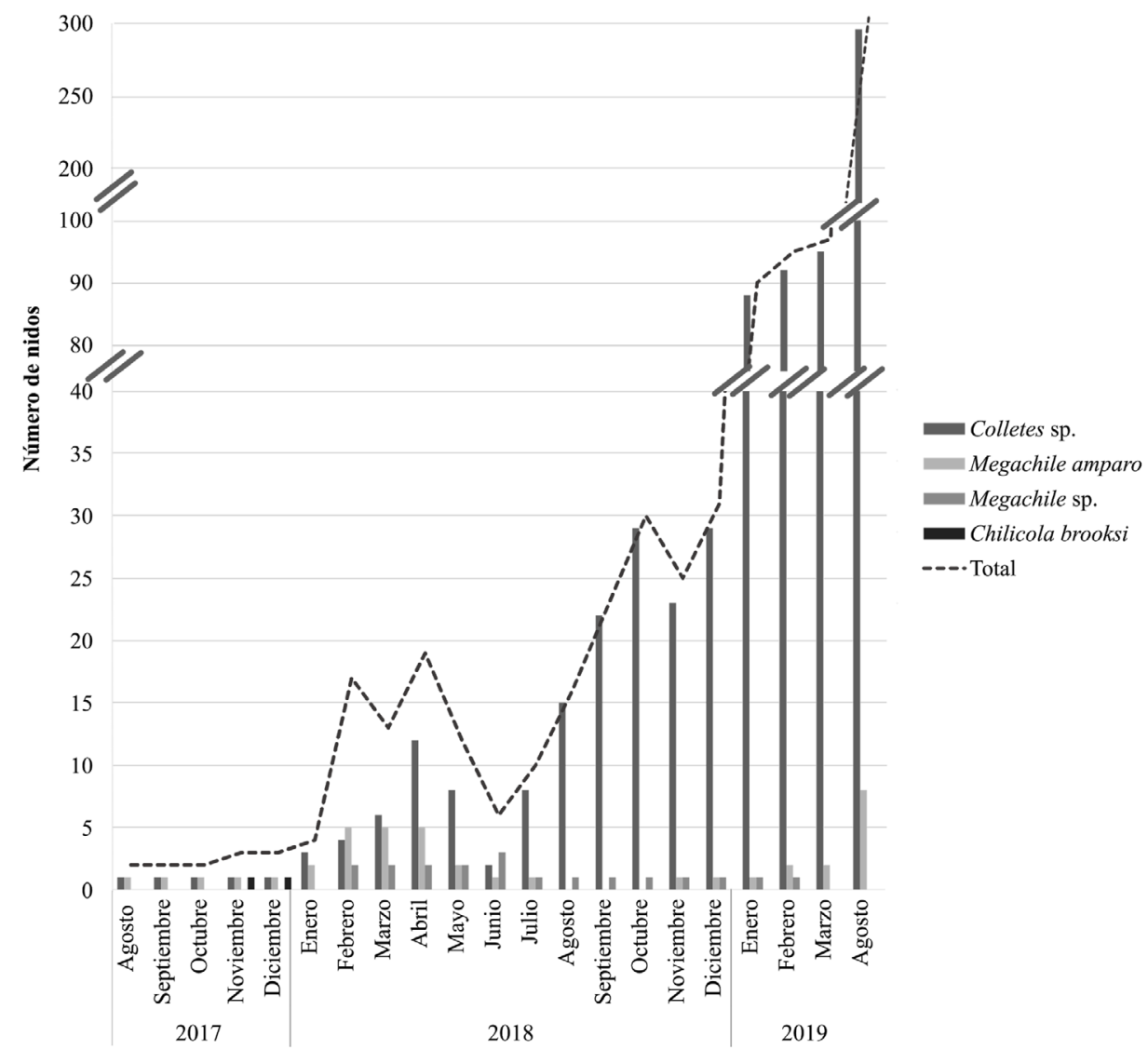

Figura 5. Comportamiento temporal de la colonización por mes y por especie de abeja para el período 2017-2019. Las barras representan el conteo neto al final de cada mes, no se tiene en cuenta la reutilización de nidos. Nótese el aumento en la línea discontinua después de junio de 2018, cuando se dejaron únicamente los materiales exitosos permitiendo un aumento significativo de nidos de Colletes sp.
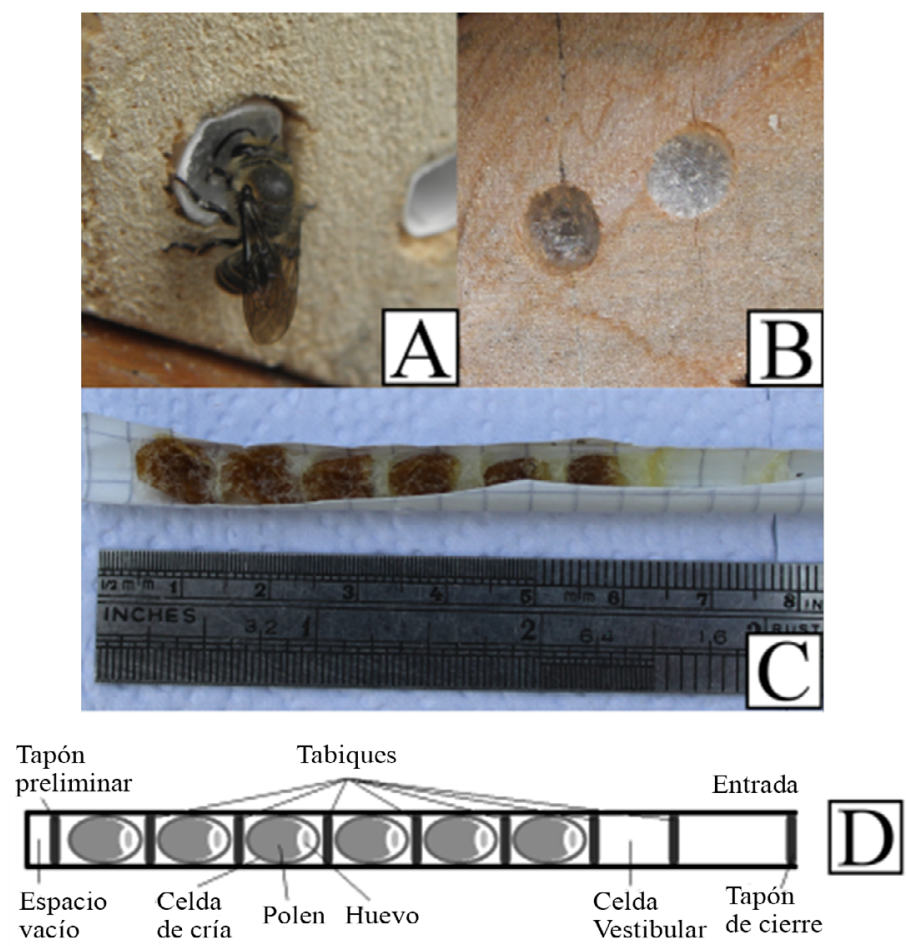

Figura 6. Arquitectura y comportamiento en nidos de Colletes sp., A. Hembra sellando un nido terminado. B. Entrada de nido sellado. C. Arquitectura interna de un nido donde se pueden observar los vestigios de las celdas de celofán y la provisión de polen. D. Diagrama de la arquitectura interna de un nido. Terminología basada en da Costa y Gonçalves (2019). 
Para M. amparo el número de celdas osciló entre dos y nueve, construidas con cortes de hojas enrolladas y superpuestas, los cortes que formaban las paredes eran elípticos o rectangulares redondeados, mientras las tapas de las celdas estaban formadas por varios cortes circulares y cóncavos. Los nidos presentaron tapón preliminar, espacio vacío antes de la primera celda y tapón de cierre. Entre celdas, generalmente, se presentaron tabiques internos en forma cóncava. No fue posible obtener mayor detalle de la arquitectura interna debido al número reducido de eventos de nidificación de esta especie. Además, el procedimiento es invasivo y se podrían lastimar las larvas e interrumpir su desarrollo. Cada celda contó con un único huevo y provisión de polen. La entrada de los nidos terminados era sellada con recortes de hojas (Fig. 7). El tamaño promedio de las celdas fue mayor en M. amparo en comparación con las celdas de Colletes sp., aunque el tamaño de las celdas de esta especie es muy variable (Tabla 1).

Durante el periodo del monitoreo también se observó la presencia de otros artrópodos que hacían uso temporal o permanente de los módulos, las más constantes fueron arañas (Araneae) 15,2 \% que aprovechaban la forma del mismo para construcción de sus telarañas y nidos. También observamos tijeretas (Dermaptera: Forficulidae) 1,6 \%, que usaban el mó-
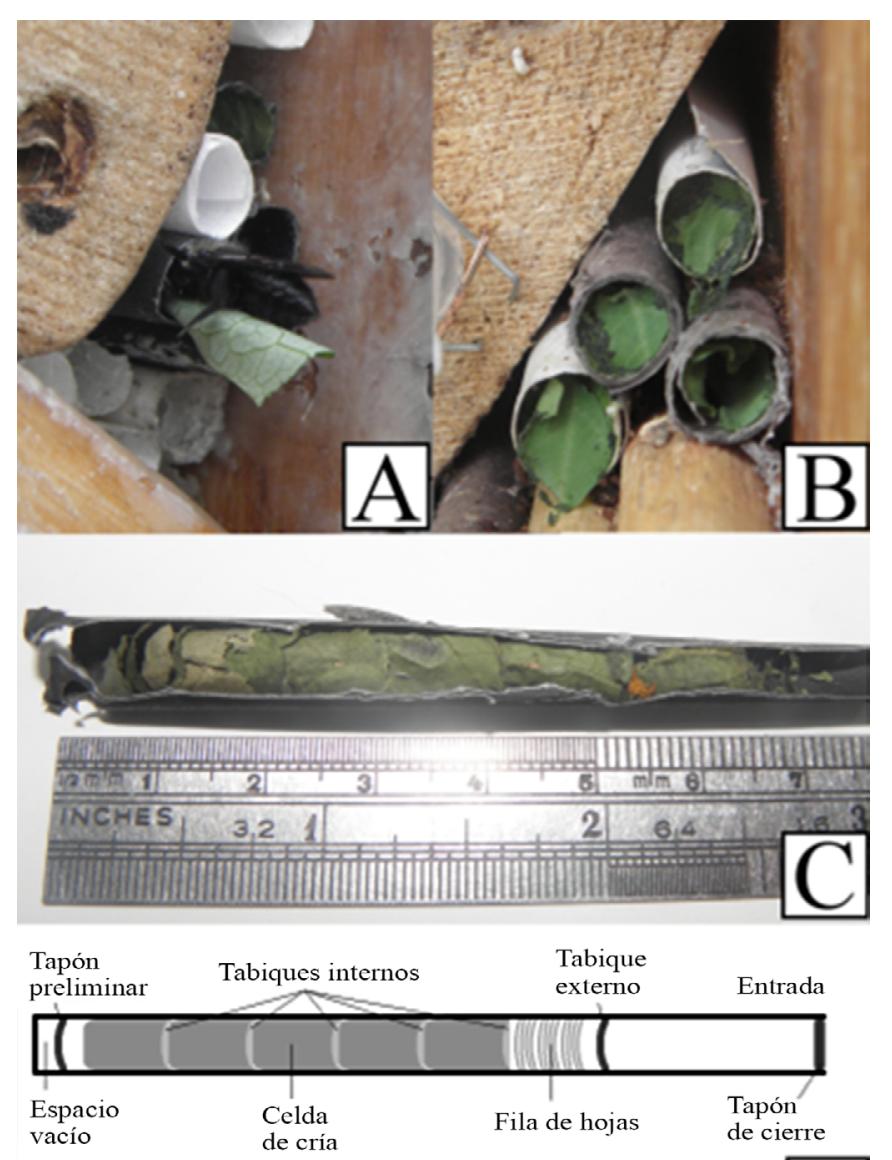

Figura 7. Arquitectura y comportamiento en nidos de M. amparo. A. Hembra entrando al nido con recortes de hojas. B. Entradas de nidos sellados. C. Arquitectura interna de nido, celdas construidas con recortes de hojas. D. Diagrama de la arquitectura interna de un nido. Terminología basada en da Costa y Gonçalves (2019).
Tabla 1. Tamaño de celdas de los nidos de abejas que colonizaron los domicilios artificiales.

\begin{tabular}{lccc}
\hline & $\begin{array}{c}\text { Colletes } \\
\text { sp. }\end{array}$ & $\begin{array}{c}\text { Megachile } \\
\text { amparo }\end{array}$ & $\begin{array}{c}\text { Chilicola } \\
\text { brooksi }\end{array}$ \\
\hline Promedio del largo de celda (mm)* & 10,02 & 12,13 & 9,99 \\
\hline Desviación estándar & 1,77 & 1,63 & 0,52 \\
\hline Min. (mm) & 6 & 8,6 & 9,62 \\
\hline Máx. (mm) & 14,54 & 15,39 & 10,35 \\
\hline Número de celdas por nido & $2-6$ & $2-9$ & 2 \\
\hline N (celdas medidas) & 31 & 50 & 2 \\
\hline N (nidos medidos) & 8 & 8 & 1 \\
\hline
\end{tabular}

* El largo total se tomó como el diámetro interno desde la base hasta el final de la celda.

dulo como refugio, y se alimentaban de los restos de los nidos y polen en descomposición. Así mismo, polillas (Lepidoptera) $0,3 \%$, grillos (Orthoptera) $0,5 \%$, moscas pequeñas (Diptera) $1,3 \%$, gorgojos (Coleoptera) 1,5\%, babosas (Pulmonata) 1,6 $\%$ y piojos de libro (Psocoptera) 2,5\%, que se observaron refugiados entre cavidades de los materiales. Algunos himenópteros (Pompilidae) 2,7\%, usaron materiales como pitillos de papel y espacios entre los tubos y paredes de los módulos para construir sus nidos de barro.

Recursos florales. Se encontraron un total de 37 tipos polínicos al interior de las muestras de nidos de las tres especies de abeja: Colletes sp., M. amparo y C. brooksi (Fig. 8), se leyeron cinco muestras de polen de un nido de C. brooksi, 18 de M. amparo y 27 de Colletes sp. En las muestras de Colletes sp., el palinomorfo predominante fue Monnina sp., presentándose además con abundancias superiores al $80 \%$ en todos los casos. Otros palinomorfos de menor abundancia y frecuencia fueron un morfotipo de Asteraceae en el $11 \%$ de las muestras, y otro morfotipo indeterminado en el $7 \%$ de las muestras. Para M. amparo, se encontraron 30 palinomorfos, siendo el más frecuente en las muestras Monnina sp., aunque en bajas abundancias, seguida en frecuencia por dos morfotipos indeterminados. Los palinomorfos más abundantes dentro de las muestras correspondieron a Tropaeolum majus L. (Tropaeolaceae), y dos más indeterminados con abundancias por encima del $50 \%$ en la mayoría de las muestras. Para las muestras de C. brooksi se encontraron seis palinomorfos, siendo los más abundantes y frecuentes tres morfotipos de Asteraceae. En la Figura 9 se ilustran algunos de los tipos polínicos más frecuentes.

Divulgación y experiencias de educación. Los domicilios artificiales han sido objeto de investigaciones con resultados presentados en un proyecto editorial institucional (Rodríguez-C. et al. 2019) y distintos espacios académicos, además ha despertado el interés de la ciudadanía. Hacia mediados de 2019 se registró la mayor cantidad de solicitudes de información, consultas y entrevistas ofrecidas a diferentes medios de comunicación, estas interacciones se resumen en la Tabla 2. 


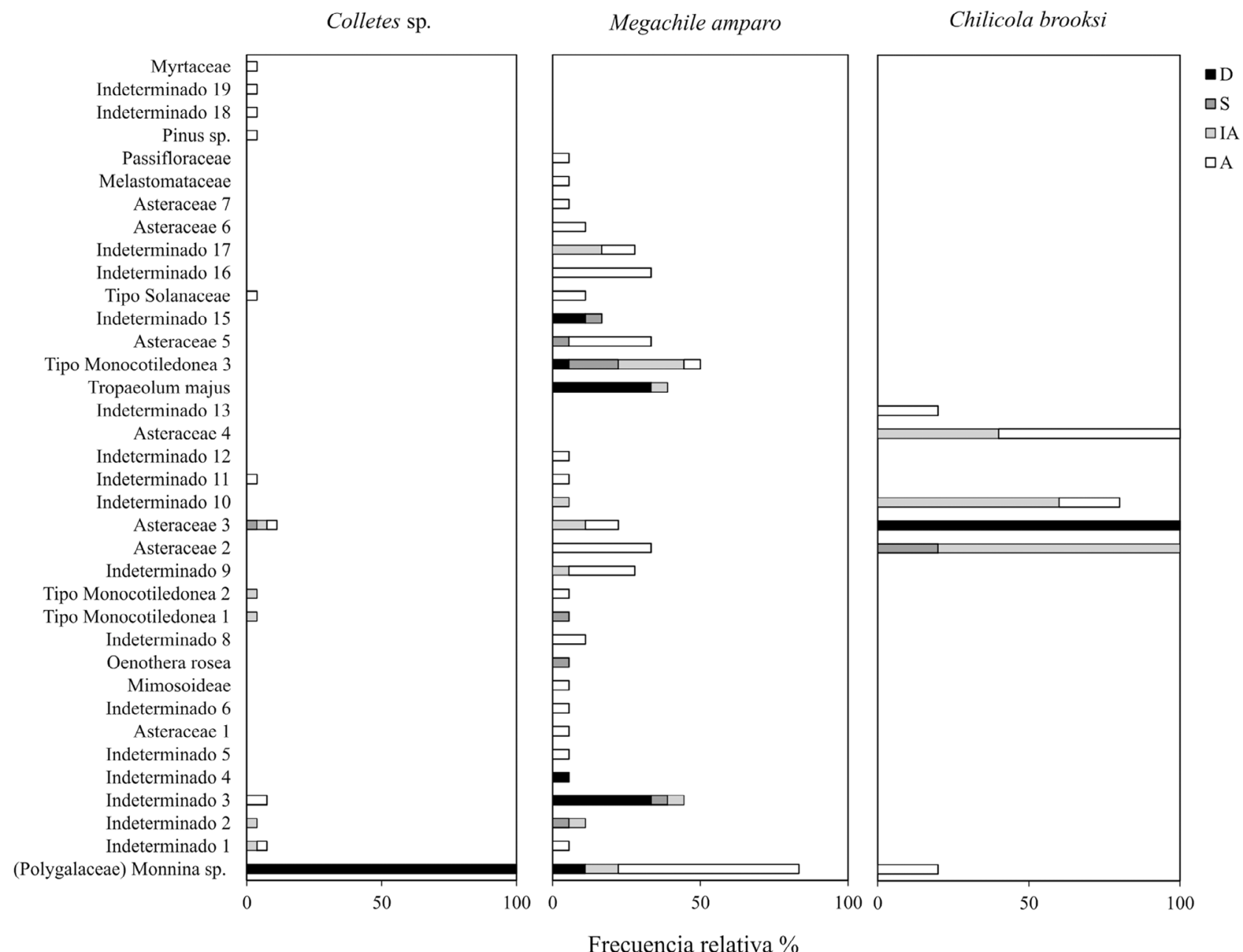

Figura 8. Frecuencias y abundancias de los palinomorfos encontrados en nidos de abejas habitantes de domicilios artificiales. D: Dominante (presente en las muestras por encima del $45 \%$ ), S: Secundario (Entre 45 y $15 \%$ del total de granos contados), IA: Importante- Aislado (entre 15 y $3 \%$ ), A: Aislado (presente en $<3 \%$ de los granos contados).

Siguiendo la clasificación jerárquica de SE propuesta por Haines-Young y Potschin (2013), se encontró que esta experiencia hizo aportes en los siguientes aspectos específicos de los SE culturales: a. interacción experiencial, con la posibilidad de que los visitantes del Jardín observen directamente a las abejas nidificando y llegando a los nidos con los recursos vegetales que recolectaban; b. científico, por la posibilidad de realizar observaciones y obtener datos del comportamiento de abejas de las que se tiene escasa información; c. educativo, uso del módulo por parte de personal de la institución para explicar conceptos biológicos a público general y estudiantes interesados; d. entretenimiento, con las publicaciones de medios de comunicación masiva que mostraron interés en divulgar la experiencia; e. estética, añadiendo elementos de disfrute para los visitantes que recorren el Jardín Botánico y finalmente, f. otros, aporte de ejemplares a colecciones entomológicas especializadas como la del Laboratorio de investigaciones en abejas de la Universidad Nacional de Colombia (LABUN) para su estudio.

\section{Discusión}

De las 16 especies de abejas conocidas para la zona (Rodríguez-C et al. 2019) solo tres se observaron nidificando en los domicilios instalados, además se observaron tres eventos de nidificación de una especie del género Megachile con patrones de coloración diferente a $M$. amparo, por lo que fue tomada como una especie diferente. Esto indica que los módulos fueron exitosos ya que los materiales disponibles fueron ocupados, no obstante, un gran porcentaje ( $81 \%$ ) de las abejas silvestres encontradas en el Jardín Botánico corresponde a abejas que utilizan otro tipo de sustratos como el suelo o cavidades verticales. Para estas especies se hace necesario desarrollar otro tipo de métodos que permitan realizar seguimiento a su biología reproductiva y al comportamiento de nidificación (Cane 2015).

La zona de páramo y bosque alto andino dentro del Jardín Botánico es un espacio que agrupa una gran cantidad de plantas nativas que recrean dichos ecosistemas, con individuos de especies de los géneros Monnina Ruiz \& Pav. (Polygalaceae), Puya Molina (Bromeliaceae), Lupinus L. (Fabaceae), Weinmannia L. (Cunoniaceae), Escallonia R. Br. ex Dumort. (Escalloniaceae), Polylepis Ruiz \& Pav. (Rosaceae), Rubus L. (Rosaceae) y algunas representantes de la familia Asteraceae como Montanoa quadrangularis Sch. Bip. ex C. Koch, Hypochaeris radicata L., Taraxacum officinale L., Baccharis spp., Sonchus oleraceus L., entre otros, que se encuentran frecuentemente florecidos. Es además una zona que recibe poco tratamiento con plaguicidas, herbicidas, tiene irrigación por aspersión y flujo de visitantes por senderos establecidos. Es- 


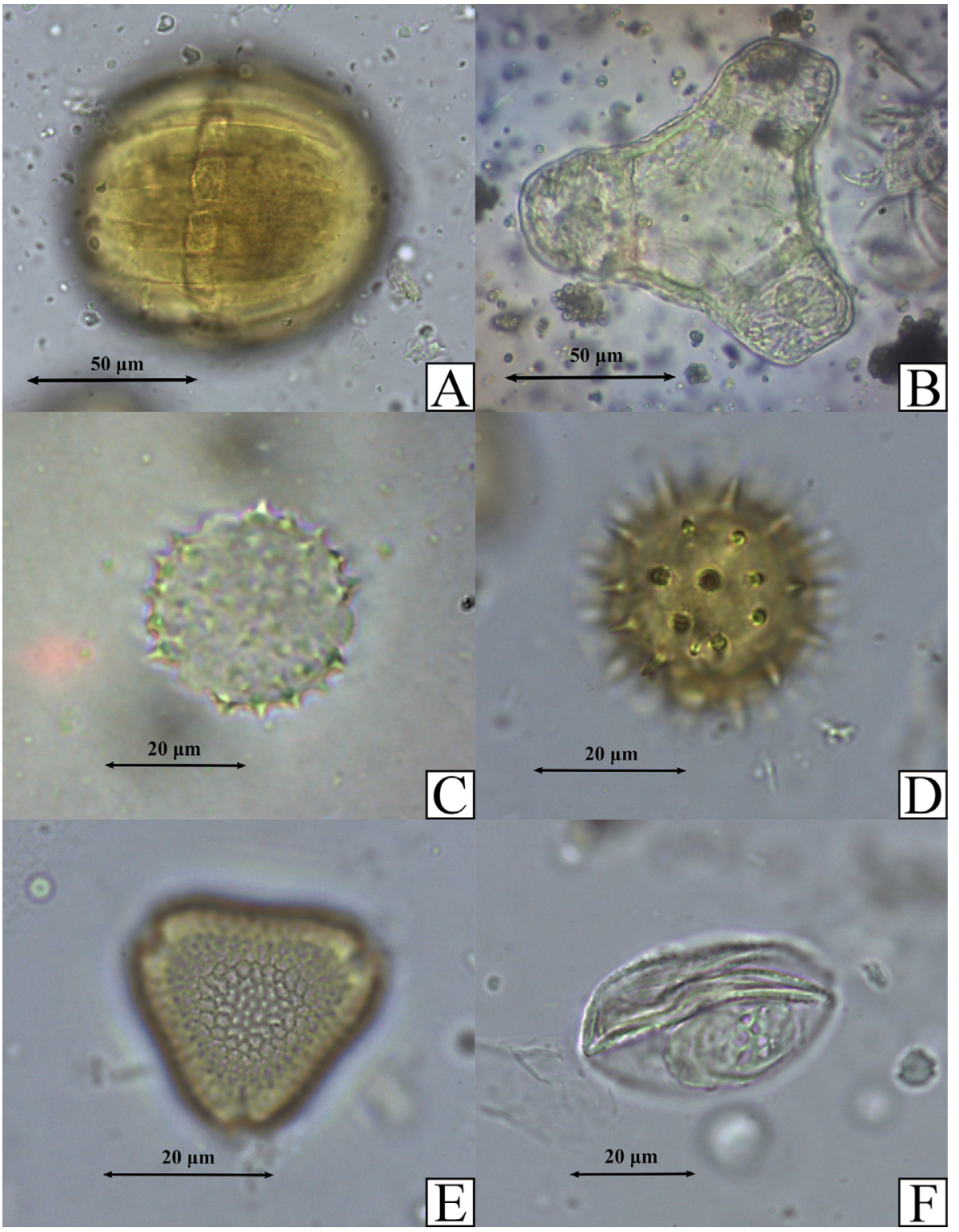

Figura 9. Palinomorfos más frecuentes en las muestras de las especies nidificadoras en los domicilios. A. Monnina sp. en vista ecuatorial. B. Oenothera rosea Aiton (Onagraceae) en vista polar C. Asteraceae 3 (indeterminada) en vista polar. D. Asteraceae 5 (indeterminada) en vista polar. E. Tropaeolum majus en vista polar. F. Tipo monocotiledonea 3 (indeterminada) en vista ecuatorial.

Tabla 2. Resumen de actividades de divulgación y difusión.

\begin{tabular}{lccl}
\hline \multicolumn{1}{c}{ Tipo de medio } & Cantidad & $\begin{array}{l}\text { Tipo de } \\
\text { público }\end{array}$ & \multicolumn{1}{c}{ Fuente } \\
\hline Académico escrito & 2 & $\begin{array}{l}\text { General y } \\
\text { Científico }\end{array}$ & $\begin{array}{l}\text { Libro: Rodríguez-C. et al. (2019) } \\
\text { Artículo científico: esta publicación }\end{array}$ \\
\hline Evento académico nacional & 3 & Científico & $\begin{array}{l}\text { Rodríguez et al. (2016), Rodríguez-C. y García-M. (2018), } \\
\text { Currea-M. y Rodríguez (2019) }\end{array}$ \\
\hline Evento académico internacional & 1 & Científico & Rodríguez-C. (2018) \\
\hline Medios informativos digitales & 9 & General & $\begin{array}{l}\text { Canal Capital (2019), China Xinhua Español (2019), El } \\
\text { Espectador (2019), Estupiñán (2019), JBB (2019), La Nota } \\
\text { Positiva (2019), Martin-L. (2019), Puentes (2019), Tvagro } \\
\text { (2019) }\end{array}$ \\
\hline Consultas atendidas & 10 & $\begin{array}{c}\text { General } \\
\text { interesado en } \\
\text { el tema espe- } \\
\text { cífico }\end{array}$ & $\begin{array}{l}\text { Registro de Sistema Distrital de Quejas y Soluciones del } \\
\text { Jegistro de visitantes atendidos del Herbario JBB con tema } \\
\text { de consulta hoteles de abejas, polinización por abejas. }\end{array}$ \\
\hline
\end{tabular}


tas pueden ser algunas de las razones que permitieron que la nidificación de abejas en el módulo de esta zona fuera exitosa en comparación con las otras dos zonas, donde se presentaba constante manejo y perturbación por riego, podas y rotación, además de mayor cantidad de reportes de saqueo de material y vandalismo.

Da Costa y Gonçalves (2019), en su revisión de trabajos con nidos trampa en el neotrópico reportaron la escasez de estudios en Colletidae y sugirieron la realización de más estudios para grupos de Megachilidae dado que son relativamente frecuentes en este tipo de nidos, siendo justamente los grupos de los que se obtuvo más información en este trabajo. Los tiempos de emergencia observados pueden corresponder a la duración del ciclo de desarrollo de estas especies, aunque para tener certeza se requieren más réplicas y observaciones detalladas. Este y otros aspectos de la biología fueron explorados de manera preliminar en este trabajo, pero se recomienda ampliar el alcance y duración para futuras investigaciones en estas especies.

De los materiales evaluados, los que fueron mayormente utilizados se caracterizaron por tener diámetros por encima de los $5 \mathrm{~mm}$, esta característica ha sido recomendada por otros autores a la hora de instalar hoteles para abejas (MacIvor 2017). Igualmente, la elección del material del nido para $M$. amparo corresponde a lo reportado para otras especies de Megachilidae (MacIvor 2017). Este tipo de abejas prefieren cavidades con entradas de tamaños entre 6 y $10 \mathrm{~mm}$, con materiales que respiren como el papel y la madera (Kim 1992; Sabino y Antonini 2011). Existe en la literatura poca información sobre la biología reproductiva de M. amparo (González 2006), por lo que todo lo encontrado en esta investigación es un primer aporte al conocimiento de esta especie y su biología.

Para el caso de Colletes sp., el trabajo con este diseño de nidos artificiales permitió observar el comportamiento de nidificación de esta especie de la que no se tenían reportes de su presencia y biología para Bogotá, con el uso de materiales económicos y fáciles de usar como los tubos de papel, que han sido evaluados en estudios similares (MacIvor 2017). Así mismo, las características de las celdas y la arquitectura del nido corresponden con lo reportado por otros autores para otras especies del género Colletes que construyen series lineares de celdas de secreciones glandulares y que nidifican en cavidades preexistentes como tallos de plantas (Almeida 2008).

El comportamiento temporal en el número de nidos, indica que las hembras de Colletes sp. llegan a tener un comportamiento dominante a la hora de elegir cavidades para nidificar, por lo que es posible que desplacen otras especies como $M$. amparo, que presentó disminución significativa en la segunda fase del estudio. Este comportamiento también puede indicar, como lo han reportados otros autores para especies de Colletes, que las hembras suelen nidificar cerca de nidos de otras hembras de la misma especie, formando agregaciones (Torchio 1965; Batra 1978).

A pesar de que en condiciones naturales se observaron hembras de Colletes sp. que nidificaban en tallos de Monnina sp., los tallos de esta especie en el módulo no fueron usados en la proporción esperada. Sin embargo, Colletes sp. tiene una estrecha relación con esta especie como recurso floral. Su frecuencia y abundancia en las muestras de polen, puede estar indicando un caso de comportamiento oligoléctico, aunque se observaron otros palinomorfos, que nunca fueron contados en la misma frecuencia y proporción. Este comportamiento ya ha sido reportado para otras especies de la misma familia (Schlindwein y Wittmann 1997; Gaglianone 2000) e incluso del género (Müller y Kuhlmann 2003).

En el caso de $M$. amparo, su comportamiento y arquitectura de nido es similar a algunas especies de su género (Kim 1992; Marinho et al. 2018). De igual forma, M. amparo eligió materiales con diámetros mayores a los de Colletes sp. y que simulan cavidades oscuras, además escogieron nidos en las esquinas de los tratamientos del módulo donde las entradas de los nidos tenían más sombra, como lo reportan Sabino y Antonini (2011). Respecto a los recursos florales esta especie se puede considerar poliléctica, ya que dentro de un mismo nido las celdas pueden ser aprovisionadas con polen de diferente origen botánico con diferentes abundancias. De los palinomorfos identificados tanto T. majus como $O$. rosea, son plantas que se encuentran frecuente y abundantemente dentro del Jardín. Otros autores también han reportado la familia Onagraceae, como un recurso importante para otras especies de Megachile (Buschini et al. 2009). Respecto a C. brooksi a pesar de que el número de celdas y de muestras de polen que se pudo analizar fue muy poco, en todas las muestras se encontraron diferentes morfotipos de Asteraceae de forma abundante, la relación con esta familia ha sido reportada para otras especies del género e incluso para el mismo subgénero, que no solo la usan como recurso floral, sino que suelen anidar en tallos huecos de diferentes especies de esta familia (Michener 2002; González y Giraldo 2009).

En paralelo con la fase de investigación, se dieron varios eventos de divulgación y educación ambiental en torno al uso de los domicilios artificiales para abejas. El número de personas interesadas y de medios de comunicación que contactaron a las autoras con el fin de conocer más acerca de esta experiencia, aumentó en la última fase de la investigación lo que demostró mayor interés en conocer acerca de las abejas locales y de las herramientas que permiten conocerlas. En espacios como los jardines botánicos las herramientas de visualización para explicar al público procesos como la polinización traen ventajas para la interacción con los visitantes (Li et al. 2020), estos módulos permiten ofrecer al ciudadano un elemento real, más allá de las palabras abstractas para explicar la presencia e importancia de polinizadores de una manera didáctica y segura. Por la naturaleza divulgativa de este trabajo, se constituye en un aporte a la línea de educación, divulgación y participación comunitaria del plan de acción de la Iniciativa Colombiana de Polinizadores capítulo Abejas, ICPA (Nates-Parra 2017a).

Con base en la experiencia en el Jardín Botánico de Bogotá se pueden dar las siguientes recomendaciones generales para trabajos similares que quieran emprenderse en otros jardines botánicos o espacios urbanos:

- Obtener conocimiento previo de las especies presentes o reportadas para la zona de interés por medio de observación directa y consultas a académicos, a instituciones dedicadas al estudio de la biodiversidad, a plataformas colaborativas de ciencia ciudadana para identificar fotografías y en literatura.

- Preferir estrategias de gestión de coberturas verdes a nivel local y regional, la siembra de recursos alimenticios y mantenimiento de zonas con sustratos de nidificación naturales diversos antes de establecer instalaciones en "desiertos" para las abejas.

- Evaluar el potencial de los domicilios artificiales y los modelos a utilizar para lograr favorecer la mayor 
cantidad de especies y no desestabilizar las poblaciones existentes.

- Utilizar materiales que sean fáciles de obtener, no contaminantes, que incluyan o imiten los usados naturalmente en la región de la instalación y que no generen condiciones adversas de humedad y temperatura a sus hospederos.

- Procurar tamaños de módulos pequeños o medianos con el fin de facilitar el mantenimiento y seguimiento.

- Proteger los módulos del exceso de sol y lluvia con techos para evitar que los materiales se deterioren o pudran.

- Instalarlos en zonas alejadas de olores fuertes, evitar la cercanía de tránsito de personas y de sustancias contaminantes como agroquímicos.

- Asegurar el monitoreo y mantenimiento periódico del módulo.

- Garantizar la provisión constante de material en buenas condiciones.

- Mantener una reserva de materiales para reemplazar el deteriorado.

- Asegurar la provisión de alimento variado y constante en el año con las plantas de la zona.

- Señalizar con información que pueda ser comprendida por cualquier observador.

- Evitar promover la comercialización masiva de estructuras sin evaluar su verdadero aporte o pertinencia.

- Incentivar espacios de educación y acercamiento del público a la función de dichas estructuras y de las abejas que las habitan.

\section{Conclusiones}

Los domicilios artificiales para abejas son herramientas útiles que facilitan la investigación en biología y comportamiento de abejas nativas solitarias que nidifican en cavidades preexistentes en zonas urbanas.

Mediante esta investigación se obtuvo información sobre recursos florales y preferencias de nidificación de especies de abejas que pese a encontrarse en un área urbana de fácil acceso, no contaban con datos sobre su biología.

Los servicios culturales prestados por abejas pueden potenciarse con este tipo de módulos ya que permiten interacciones experienciales con los ciudadanos con el fin de disminuir la entomofobia y aumentar el interés acerca de las abejas silvestres y su importancia en los ecosistemas.

Dado que los jardines botánicos son instituciones que incluyen en sus actividades misionales la investigación y la educación ambiental, no sólo de las plantas sino de sus relaciones con otros organismos, son espacios idóneos para implementar iniciativas como las del presente estudio.

\section{Agradecimientos}

Las autoras agradecemos a los profesionales del Jardín Botánico José Celestino Mutis que apoyaron la presente investigación, a los tesistas y pasantes que ayudaron en la instalación y revisión de los módulos. A Víctor Hugo González por la determinación de ejemplares recolectados durante el proyecto. Al Laboratorio de Investigaciones en Abejas de la Universidad Nacional de Colombia (LABUN) por permitir el uso de equipos para el análisis palinológico, a José Muñoz por su apoyo en la elaboración de los mapas y a los evaluadores del manuscrito por sus valiosos aportes.

\section{Literatura citada}

ALLASINO, M. L.; MARRERO, H. J.; DORADO, J.; TORRETTA, J. P. 2019. Scientific note: first global report of a bee nest built only with plastic. Apidologie 50 (2): 230-233. https://doi. org/10.1007/s13592-019-00635-6

ALMEIDA, E. A. B. 2008. Colletidae nesting biology (Hymenoptera: Apoidea). Apidologie 39 (1): 16-29. https://doi.org/10.1051/ apido:2007049

AMEIXA, O. M. C. C.; SOARES, A. O.; SOARES, A. M. V. M.; LILLEBØ, A. I. 2018. Ecosystem services provided by the little things that run the world. pp. 267-302. In: Şen, B.; Grillo, O. (Eds.). Selected studies in biodiversity. IntechOpen. Londres, Reino Unido. 400 pp. http://dx.doi.org/10.5772/ intechopen. 74847

BATRA, S. W. T. 1978. Aggression, territoriality, mating and nest aggregation of some solitary bees (Hymenoptera: Halictidae, Megachilidae, Colletidae, Anthophoridae). Journal of the Kansas Entomological Society 51 (4): 547-559. https://www.jstor. org/stable/25083851

BAUER, E. C.; LYNCH, L. I.; GOLICK, D. A.; WEISSLING, T. J. 2015. Creating a solitary bee hotel. Neb Guide, University of Nebraska- Lincoln Extension G2256. Disponible en: https:// entomology.unl.edu/scilit/Creating\%20a\%20Solitary\%20 Bee\%20Hotel.pdf [Fecha revisión: 10 septiembre 2019]

BGM, BOTANISCHER GARTEN MÜNCHEN-NYMPHENBURG. 2019. Bees and bumble bees: Munich Botanical Garden. Disponible en: https://www.botmuc.de/en/bees/. [Fecha revisión: 19 diciembre 2019]

BONILLA, M. A. 2017. Capítulo 2: El servicio ecosistémico de polinización prestado por abejas. pp. 41-58. En: Nates-Parra, G. (Ed.). Iniciativa Colombiana de Polinizadores - Abejas - ICPA. Universidad Nacional de Colombia. Bogotá, Colombia. 362 pp. https://www.uneditorial.com/pageflip/acceso-abierto/pdf/ abejas-polinizadoras-ebook-40217.pdf

BUSCHINI, M. L. T.; RIGON, J.; CORDEIRO, J. 2009. Plants used by Megachile (Moureapis) sp. (Hymenoptera: Megachilidae) in the provisioning of their nests. Brazilian Journal of Biology 69 (4): 1187-1194. https://www.scielo.br/scielo.php?script=sci_ arttext\&pid=S1519-69842009000500025.

CANAL CAPITAL. 2019. Hoteles para abejas en el Jardín Botánico. Disponible de Capital Noticias en: https:/conexioncapital.co/ hoteles-para-abejas-jardin-botanico/. [Fecha revisión: 13 julio 2020].

CANE, J. H. 2015. Landscaping pebbles attract nesting by the native ground-nesting bee Halictus rubicundus (Hymenoptera: Halictidae). Apidologie 46 (6): 728-734. https://doi.org/10.1007/ s13592-015-0364-z

CARLTON, M. 2017. How to make and manage a bee hotel: instructions that really work. The Pollinator Garden. Disponible en: https://www.foxleas.com/make-a-bee-hotel.asp. [Fecha revisión: 7 noviembre 2019]

CHINA XINHUA ESPAÑOL. 2019. Hoteles para abejas, iniciativa para preservar a los polinizadores del planeta. Disponible en: https://www.youtube.com/watch? $v=i 4 n S R-$ P4EaM\&feature=youtu.be . [Fecha revisión: 13 julio 2020].

CURREA-M., S.; RODRÍGUEZ, Á. 2019. Jardines botánicos, más que zonas verdes: custodios de la biodiversidad urbana, caso abejas en Bogotá. pp. 32-33. En: Jaramillo, M. A.; Silva-Tamayo, J. C.; Waldron, T. El papel de la naturaleza en nuestro bienestar: Memorias I Congreso Colombiano de Servicios Ecosistémicos, Universidad Militar Nueva Granada. Cajicá, Colombia. 71 pp. https://510574da-8926-4864-b3b9-47fb718790dd.filesusr.com/ ugd/d02af5_9f587a04472a4771ad9088a744d8502e.pdf

DA COSTA, C. C. F. DA; GONÇALVES, R. B. 2019. What do we know about Neotropical trap-nesting bees? Synopsis about their nest biology and taxonomy. Papéis Avulsos de Zoologia 59: e20195926. https://dx.doi.org/10.11606/1807-0205/2019.59.26 
DEAN, R. 2010. Information sheet bee hotels. Disponible en: https:// bwars.com/sites/www.bwars.com/files/info sheets/13 Bee Hotels_20120217_infosheet.pdf [Fecha revisión: 22 octubre 2019].

EL ESPECTADOR. 2019. En imágenes: así es el hotel de abejas que hay en Bogotá. Disponible en: https://www.elespectador.com/ noticias/bogota/en-imagenes-asi-es-el-hotel-de-abejas-que-hayen-bogota-galeria-889876/ . [Fecha revisión: 13 julio 2020].

ESTUPIÑÁN, K. 2019. Conoce los 'hoteles' para abejas que tiene el Jardín Botánico en Bogotá. Disponible de la Alcaldía de Bogotá D.C. Disponible en: https://bogota.gov.co/mi-ciudad/ambiente/ dia-mundial-de-las-abejas . [Fecha revisión: 13 julio 2020].

FONNEGRA, R. 1989. Métodos de estudio palinológico. Universidad de Antioquia, Centro de Investigaciones de la Facultad de Ciencias Naturales y Exactas. Medellin, Colombia. $57 \mathrm{pp}$.

FORTEL, L.; HENRY, M.; GUILBAUD, L.; MOURET, H.; VAISSIÈRE, B. E. 2016. Use of human-made nesting structures by wild bees in an urban environment. Journal of Insect Conservation 20 (2): 239-253. https://doi.org/10.1007/s10841016-9857-y

GAGLIANONE, M. C. 2000. Behavior on flowers, structures associated to pollen transport and nesting biology of Perditomorpha brunerii and Cephalurgus anomalus (Hymenoptera: Colletidae, Andrenidae). Revista de Biología Tropical 48 (1): 89-99. https://www.scielo.sa.cr/scielo.php? pid=S 0034 $77442000000100010 \&$ script $=$ sci_arttext\&tlng=en

GARDINER, T.; FARGEAUD, K. 2018. Build it and they will come: Grasshoppers check-in to a grassland bee hotel. Journal of Orthoptera Research 27 (2): 159-161. https://doi.org/10.3897/ jor. 27.28385

GATHMANN, A.; GREILER, H. J.; TSCHARNTKE, T. 1994. Trapnesting bees and wasps colonizing set-aside fields: succession and body size, management by cutting and sowing. Oecologia 98 (1): 8-14. https://doi.org/10.1007/BF00326084

GIRALDO, C.; RODRÍGUEZ, A.; CHAMORRO, F. J.; OBREGÓN, D.; MONTOYA, P.; RAMÍREZ, N.; SOLARTE, V.; NATESPARRA, G. 2011. Guía ilustrada de polen y plantas nativas visitadas por abejas: Cundinamarca, Boyacá, Santander, Sucre, Atlántico y Sierra Nevada de Santa Marta, Colombia. Bogotá: Universidad Nacional de Colombia. Bogotá, Colombia. 230 pp.

GONZÁLEZ, V. H. 2006. Dos especies nuevas de abejas (Hymenoptera) de la ciudad de Bogotá (Colombia). Revista Colombiana de Entomología 32 (1): 93-96. http://www.scielo. org.co/pdf/rcen/v32n1/v32n1a15.pdf.

GONZÁLEZ, V. H.; GIRALDO, C. 2009. New Andean bee species of Chilicola Spinola (Hymenoptera: Colletidae, Xeromelissinae) with notes on their biology. Caldasia 31 (1): 145-154. http:// dx.doi.org/10.15446/caldasia.

GONZÁlEZ, V. H.; OSPINA, M.; BENNETT, D. 2005. Abejas altoandinas de Colombia: Guía de campo. Instituto de Investigación de Recursos Biológicos Alexander von Humboldt. Bogotá, Colombia. 80 pp. http://repository. humboldt.org.co/bitstream/handle/20.500.11761/31359/74. pdf? sequence $=1$ \&isAllowed $=y$

HAINES-YOUNG, R.; POTSCHIN, M. 2013. Common International Classification of Ecosystem Services (CICES): Consultation on Version 4, August-December 2012. EEA Framework Contract No EEA/IEA/09/003. Disponible en: https://cices.eu/ content/uploads/sites/8/2012/07/CICES-V43_Revised-Final_ Report 29012013.pdf [Fecha revisión: 21 octubre 2020]

HALCROFT, M. 2012. How to make nests for Reed bees - Bundles. Disponible en: https://www.beesbusiness.com.au/articles/ How\%20to\%20make\%20nests\%20for\%20reed\%20bees\%20 -\%20bundles.pdf. [Fecha revisión: 22 octubre 2019]

HALL, D. M.; CAMILO, G. R.; TONIETTO, R. K.; OLLERTON, J.; AHRNÉ, K.; ARDUSER, M.; ASCHER, J. S.; BALDOCK, K. C. R.; FOWLER, R.; FRANKIE, G.; GOULSON, D.; GUNNARSSON, B.; HANLEY, M. E.; JACKSON, J. I.;
LANGELlOTTO, G.; LOWENSTEIN, D.; MINOR, E. S.; PHILPOTT, S. M.; POTTS, S. G.; SIROHI, M. H.; SPEVAK, E. M.; STONE, G. N.; THRELFALL, C. G. 2017. The city as a refuge for insect pollinators. Conservation Biology 31 (1): 2429. https://doi.org/10.1111/cobi.12840

JBB, JARDÍN BOTÁNICO DE BOGOTÁ. 2019. Jardín Botánico de Bogotá, pionero en la implementación de hoteles para abejas en la capital. Disponible en: http://www.jbb.gov.co/ index.php/noticias-del-jardin/item/322-jardin-botanicopionero-en-la-implementacion-de-hoteles-para-abejas-en-lacapital . Fecha revisión: 13 julio 2020].

KAMEL, S. M.; ABU HASHESH, T. A.; OSMAN, M. A.; SHEBL, M. A. 2007. A new model of polystyrene foam for renesting leafcutting bees (Megachile spp: Megachilidae, Hymenoptera). Agricultural Research Journal, Suez Canal University 7 (2): $97-$ 101. https://www.researchgate.net/publication/236115710_A_ new_model_of_polystyrene_foam_for_renesting_leafcutting_bees_Megachile_spp_Megachilidae_Hymenoptera

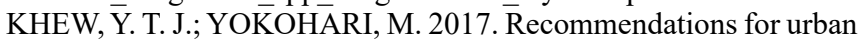
biodiversity conservation in the context of landscape preference in Singapore. Cities and the Environment 10 (1): article 4. https:// digitalcommons.lmu.edu/cate/vol10/iss $1 / 4$

KIM, J. Y. 1992. Nest dimensions of two leaf-cutter bees (Hymenoptera: Megachilidae). Annals of the Entomological Society of America 85 (1): 85-90. https://doi.org/10.1093/aesa/85.1.85

KLEIN, A. M.; VAISSIERE, B. E.; CANE, J. H.; STEFFANDEWENTER, I.; CUNNINGHAM, S. A.; KREMEN, C.; TSCHARNTKE, T. 2006. Importance of pollinators in changing landscapes for world crops. Proceedings of the Royal Society B: Biological Sciences 274 (1608): 303-313. http://doi.org/10.1098/ rspb.2006.3721

KUMAR V.; BELAVADI, V. V.; GUPTA, A. 2015. Parasitisation of leaf-cutter bees (Megachilidae: Apoidea) by Melittobia. Entomon 40(2): 103-110. http://entomon.in/index.php/Entomon/ article/view/69

LA NOTA POSITIVA. 2019. En el Jardín Botánico de Bogotá se estrenaron los primeros 'hoteles' para las abejas. Disponible en: https://lanotapositiva.com/actualidad/en-el-jardin-botanicode-bogota-se-estrenaron-los-primeros-hoteles-para-lasabejas_15104. [Fecha revisión: 13 julio 2020].

LANGELLOTTO, G. A.; MELATHOPOULOS, A.; MESSER, I.; ANDERSON, A.; MCCLINTOCK, N.; COSTNER, L. 2018. Garden pollinators and the potential for ecosystem service flow to urban and peri-urban agriculture. Sustainability 10 (6): 2047. https://doi.org/10.3390/su10062047

LI, S.; WANG, Q.; WANG, T.; ZHANG, W. 2020. Visualisation increases the public understanding and awareness of pollination in the botanical garden. Roots, Pollination and Botanic Gardens 17 (1): 39-42. https://www.bgci.org/wp/wp-content/ uploads/2020/05/Roots17.1MerRes.pdf

MACIVOR, J. S. 2016. Wild bees in cultivated city gardens. pp. 64-77. In: Brown, S.; McIvor, K.; Snyder, E. H. (Eds.). Sowing seeds in the city: Ecosystem and municipal services. Springer, Países Bajos. 407 pp. https://doi.org/10.1007/978-94-017-7453615

MACIVOR, J. S. 2017. Cavity-nest boxes for solitary bees: a century of design and research. Apidologie 48 (3): 311-327. https://doi. org/10.1007/s13592-016-0477-z

MACIVOR, J. S.; PACKER, L. 2015. "Bee hotels" as tools for native pollinator conservation: A premature verdict?. PLoS ONE 10 (3): e0122126. https://doi.org/10.1371/journal.pone.0122126

MACIVOR, J. S.; SALEHI, B. 2014. Bee species-specific nesting material attracts a generalist parasitoid: implications for co-occurring bees in nest box enhancements. Environmental Entomology 43 (4): 1027-1033. https://doi.org/10.1603/EN13241.

MADER, E.; SPIVAK, M.; EVANS, E. 2010. Managing alternative pollinators: a handbook for beekeepers, growers, and conservationists. Sustainable Agriculture Research and Education (SARE), Natural Resource, Agriculture, and Engineering 
Service (NRAES). Ithaca, New York. $186 \mathrm{pp}$. http://www. masterbeekeeping.com/images/Alternative\%20Pollinators/ Managing_Alternative_Pollinators.pdf

MARINHO, D.; MUNIZA, D.; AZEVEDO, G. 2018. Nesting biology of three Megachile (Hymenoptera: Megachilidae) species from Eastern Amazonia, Brazil. Revista Brasileira de Entomologia 62 (2): 97-106. https://doi.org/10.1016/j.rbe.2018.03.002

MARTÍNEZ, S.; SOTO, E. A.; SANDOVAL, S.; OTERO, J. T. 2017. Distribución espacial y hábitos de nidificación de Nannotrigona mellaria (Apidae: Meliponini) en una localidad de Cali (Colombia). Acta Zoológica Mexicana 33 (2): 161$168 . \quad \mathrm{http}: / / \mathrm{www}$. scielo.org.mx/pdf/azm/v33n2/2448-8445azm-33-02-00161.pdf

MARTIN-L., C. 2019. "Hoteles" cinco estrellas para abejas. Disponible de Caracol Radio en: https://caracol.com.co/ emisora/2019/11/08/bogota/1573228426_860059.html . [Fecha revisión: 13 julio 2020].

MASON, L.; ARATHI, H. S. 2019. Assessing the efficacy of citizen scientists monitoring native bees in urban areas. Global Ecology and Conservation 17: e00561. https://doi.org/10.1016/j. gecco.2019.e00561

MAZZEO, N. M.; TORRETTA, J. P. 2015. Wild bees (Hymenoptera: Apoidea) in an urban botanical garden in Buenos Aires, Argentina. Studies on Neotropical Fauna and Environment 50 (3): 182-193. https://doi.org/10.1080/01650521.2015.1093764

MICHENER, C. D. 2000. The bees of the world. Johns Hopkins University Press. Baltimore, EEUU. 913 pp. https://jhupbooks. press.jhu.edu/title/bees-world.

MICHENER, C. D. 2002. The bee genus Chilicola in the tropical Andes, with observations on nesting biology and a phylogenetic analysis of the subgenera (Hymenoptera: Colletidae, Xeromelissinae). Scientific Papers, Natural History Museum, The University of Kansas 26: 1-47. http://hdl.handle. net $/ 1808 / 26073$

MICHENER, C. D.; MCGINLEY, R. J.; DANFORTH, B. N. 1994. The Bee Genera of North and Central America: Hymenoptera Apoidea. Smithsonian Institution, Washington, EEUU. 209 pp.

MÜLLER, A.; KUHLMANN, M. 2003. Narrow flower specialization in two European bee species of the genus Colletes (Hymenoptera: Apoidea: Colletidae). European Journal of Entomology 100: 631-635. https://www.eje.cz/pdfs/eje/2003/04/25.pdf

NATES-PARRA, G. 2017a. Iniciativa Colombiana de Polinizadores - Abejas - ICPA. Universidad Nacional de Colombia. Bogotá, Colombia. 362 pp. https://www.uneditorial.com/pageflip/ acceso-abierto/pdf/abejas-polinizadoras-ebook-40217.pdf

NATES-PARRA, G. 2017b. Capítulo 13: Uso de nidos trampa. pp. 195-199. En: Nates-Parra, G. (Ed.). Iniciativa Colombiana de Polinizadores - Abejas - ICPA. Universidad Nacional de Colombia. Bogotá, Colombia. 364 pp. https://www.uneditorial. $\mathrm{com} / \mathrm{pageflip} / \mathrm{acceso}-\mathrm{abierto} / \mathrm{pdf} / \mathrm{abejas}$-polinizadorasebook-40217.pdf

NATES-PARRA, G.; MONTOYA, P. M.; CHAMORRO, F. J.; RAMÍREZ, N.; GIRALDO, C.; OBREGÓN, D. 2013. Origen geográfico y botánico de mieles de Apis mellifera (Apidae) en cuatro departamentos de Colombia. Acta Biológica Colombiana 18 (3): 427-437. https://revistas.unal.edu.co/index.php/actabiol/ article/view/38290

NATES-PARRA, G.; PARRA, A.; RODRÍGUEZ, Á.; BAQUERO, P.; VÉLEZ, D. 2006a. Abejas silvestres (Hymenoptera: Apoidea) en ecosistemas urbanos: Estudio en la ciudad de Bogotá y sus alrededores. Revista Colombiana de Entomología 32 (1): 77-84. http://www.scielo.org.co/scielo.php? script=sci arttext\&pid=S0120-04882006000100013

NATES-PARRA, G.; RODRÍGUEZ, Á.; VÉLEZ, E. D. $2006 \mathrm{~b}$. Abejas sin aguijón (Hymenoptera: Apidae: Meliponini) en cementerios de la cordillera oriental de Colombia. Acta Biológica Colombiana 11 (1): 25-35. https://revistas.unal.edu.co/index. php/actabiol/article/view/27141
PINILLA-GALLEGO, M. S.; NIETO, V.; NATES-PARRA, G. 2016. Recurso polínico y ciclo estacional de Thygater aethiops (Hymenoptera: Apidae) en un ambiente urbano (BogotáColombia). Revista de Biología Tropical 64 (3): 1247-1257. https://doi.org/10.15517/RBT.V64I3.20909.

PUENTES, A. 2019. Los 'hoteles' para abejas que puso a funcionar el Jardín Botánico. Disponible de Redacción Bogotá, El Tiempo en: https://www.eltiempo.com/bogota/jardin-botanico-puso-afuncionar-hoteles-para-abejas-366520.[Fecha revisión: 13 julio 2020].

RAMOS, D. L.; CUNHA, W. L.; EVANGELISTA, J.; LIRA, L. A.; ROCHA, M. V. C.; GOMES, P. A.; FRIZZAS, M. R.; TOGNI, P. H. B. 2020. Ecosystem services provided by insects in Brazil: what do we really know?. Neotropical Entomology 49(6), 783794. https://doi.org/10.1007/s13744-020-00781-y

REID, W. V.; MOONEY, H. A.; CROPPER, A.; CAPISTRANO, D.; CARPENTER, S. R.; CHOPRA, K.; DASGUPTA, P.; DIETZ, T.; DURAIAPPAH, A. K.; HASSAN, R.; KASPERSON, R.; LEEMANS, R.; MAY, R. M.; MCMICHAEL, A. J.; PINGALI, P.; SAMPER, C.; SCHOLES, R.; WATSON, R. T.; ZAKRI, A. H.; SHIDONG, Z.; ASH, N. J.; BENNETT, E.; KUMAR, P.; LEE, M. J.; RAUDSEPP-HEARNE, C.; SIMONS, H.; THONELL, J.; ZUREK, M. B. 2005. Ecosystems and human well-being - Synthesis. Island Press. Washington, EEUU. 137 pp. https://www.millenniumassessment.org/documents/ document.356.aspx.pdf

RJB, REAL JARDÍN BOTÁNICO. 2017. El Real Jardín Botánico pone a disposición de los insectos un hotel para mejorar la polinización. Disponible en: http:/www.rjb.csic.es/jardinbotanico/ jardin/contenido.php?Pag $=293 \&$ tipo $=$ noticia $\& \operatorname{cod}=5919 . \quad[$ Fecha revisión: 19 diciembre 2019]

RODRÍGUEZ, Á.; GÓMEZ, C.; TRIANA, H.; REYES, M. C.; PRIETO, M.; GAMBOA, J. 2016. Nidos de abejas en el Jardín Botánico de Bogotá: una oportunidad de investigación de nuestras abejas altoandinas pp. 36. En: Parra-H., A. (Ed.). Encuentro Colombiano sobre Abejas Silvestres, libro de memorias. 8. ${ }^{\text {a }}$ Ed. Universidad Nacional de Colombia. Bogotá, D.C., Colombia. 78 pp. Disponible en: https://www.uneditorial. $\mathrm{com} /$ pageflip/acceso-abierto/pdf/encuentro-colombiano-sobreabejas-silvestres-octava-edicion-libro-de-memorias.pdf [Fecha revisión: 13 julio 2020]

RODRÍGUEZ-C., Á. T. 2018. Estrategias para conocer y conservar las abejas silvestres en Bogotá D.C. - Colombia: Iniciativas desde el Jardín Botánico José Celestino Mutis. pp. 187-190. En: Escobedo, N.; Enríquez, E.; Landaverde, P.; Martínez, O.; Armas, G.; Marroquín, A.; Hernández, L.; Medina, M.; Guzmán, M.; Zepeda, R.; Gruber, T.; Straffon, S.; Escobar, D.; Méndez C.; Casiá, Q. (Eds.). Memorias X Congreso Mesoamericano Sobre Abejas Nativas: declive de las abejas nativas y retos para su conservación. Universidad de San Carlos de Guatemala. Guatemala. 438 pp. Disponible en: https://1drv.ms/b/ s!AgZ40f3nM8ligaQYQbues4Q1RSDrvA [Fecha revisión: 13 julio 2020]

RODRÍGUEZ-C., Á. T; GARCÍA-M., V. P. 2018. Observaciones preliminares de la relación de abejas del género Colletes con plantas del género Monnina (Polygalaceae), ¿un posible caso de oligolectia?. pp. 420. En: Escobedo, N.; Enríquez, E.; Landaverde, P.; Martínez, O.; Armas, G.; Marroquín, A.; Hernández, L.; Medina, M.; Guzmán, M.; Zepeda, R.; Gruber, T.; Straffon, S.; Escobar, D.; Méndez C.; Casiá, Q. (Eds.). Memorias $X$ Congreso Mesoamericano Sobre Abejas Nativas: declive de las abejas nativas y retos para su conservación. Universidad de San Carlos de Guatemala. Guatemala. 438 pp. Disponible en: https://1drv.ms/b/s!AgZ40f3nM8ligaQYQbues4Q1RSDrvA [Fecha revisión: 13 julio 2020]

RODRÍGUEZ-C., Á. T; GÓMEZ-R., W. C.; TRIANA-P., H. D. 2019. Las abejas del Jardín Botánico de Bogotá José Celestino Mutis. Colección Mora Osejo. Serie Relación Planta Organismo 
No.1. Jardín Botánico José Celestino Mutis. Bogotá, Colombia. $152 \mathrm{pp}$.

ROUBIK, D. W.; MORENO, J. E. 1991. Pollen and Spores of Barro Colorado Island. Monographs in Systematic Botany 36. Missouri Botanical Garden. Missouri, EE.UU. 268 pp.

SABINO, W. DE O.; ANTONINI, Y. 2011. Use of trap-nests with a neotropical leaf-cutter bee Megachile (Moureapis) anthidioides (Hymenoptera: Megachilidae). Journal of the Kansas Entomological Society 84 (1): 78-79. https://doi.org/10.2317/ jkes 100321.1

SCHLINDWEIN, C.; WITTMANN, D. 1997. Micro-foraging routes of Bicolletes pampeana (Colletidae) and bee-induced pollen presentation in Cajophora arechavaletae (Loasaceae). Botanica Acta 110(2): 177-183.https://doi.org/10.1111/j.1438-8677.1997. tb00626.x

SIEMASZKO, M.; ZYCH, M. 2017. Urban ecosystems - a place for pollinators? A mini-review and social implications. Formation of Urban Green Areas 1 (14): 193-201. http://www.krastotvarka. vhost.lt/documents/2017-25.pdf

SILVA-GARNICA, D.; ARCOS-D., A. L.; GÓMEZ-D., J. A. 2006. Guía ambiental apícola. Instituto de Investigación de Recursos Biológicos Alexander von Humboldt. Bogotá, Colombia. 142 pp. http://repository.humboldt.org.co/bitstream/ handle/20.500.11761/32938/GUIA AMBIENTAL APICOLA Bogota_-Colombia.pdf? sequence $=\overline{1} \&$ isAllowed $=y$

SMITH, L.; MILLER, H. (Eds.). 2020. Roots: Pollination and botanic gardens. Vol. 17 (1). Botanic Gardens Conservation International Education Review. Botanic Gardens Conservation International (BGCI). Reino Unido. 45 pp. https://www.bgci. org/wp/wp-content/uploads/2020/05/Roots17.1MerRes.pdf

SMITH-PARDO, A. H.; VÉLEZ, R. I. 2008. Abejas de Antioquia: Guía de campo. Universidad Nacional de Colombia. Medellín, Colombia. 132 pp. https:/www.researchgate.net/ publication/236856027_Abejas_de_Antioquia-_Guia_de Campo_Field_guide_to_the bees_of_Antioquia_Colombia

STAAB, M.; PUFAL, G.; TSCHARNTKE, T.; KLEIN, A. M. 2018. Trap nests for bees and wasps to analyse trophic interactions in changing environments - A systematic overview and user guide. Methods in Ecology and Evolution 9 (11): 2226-2239. https:// doi.org/10.1111/2041-210X.13070

TELLEZ-FARFÁN, L.; POSADA-FLÓREZ, F. J. 2013. Actividad polinizadora y preferencia floral de Bombus spp. (Hymenoptera: Apidae) presentes en una cerca viva. Revista U.D.C.A Actualidad \& Divulgación Científica 16 (2): 359-367. https:// doi.org/10.31910/rudca.v16.n2.2013.908

TORCHIO, P. F. 1965. Observations on the biology of Colletes ciliatoides (Hymenoptera: Apoidea, Colletidae). Journal of the Kansas Entomological Society 38 (2): 182-187. https://www. jstor.org/stable/25083432.

TSCHARNTKE, T.; GATHMANN, A.; STEFFAN-DEWENTER, I. 1998. Bioindication using trap-nesting bees and wasps and their natural enemies: Community structure and interactions. Journal of Applied Ecology 35 (5): 708-719. https://doi.org/10.1046/ j.1365-2664.1998.355343.x

TUCKER, S. K.; GINSBERG, H. S.; ALM, S. R. 2019. Eastern carpenter bee (Hymenoptera: Apidae): Nest structure, nest cell provisions, and trap nest acceptance in Rhode Island. Environmental Entomology 48 (3): 702-710. https://doi. org/10.1093/ee/nvz032

TURO, K. J.; GARDINER, M. M. 2019. From potential to practical: conserving bees in urban public green spaces. Frontiers in Ecology and the Environment 17 (3): 167-175. https://doi. org/10.1002/fee. 2015

TVAGRO. 2019. Qué son las Abejas silvestres - TvAgro por Juan Gonzalo Ángel. Disponible en: https://www.youtube.com/ watch? $\mathrm{v}=3 \mathrm{kxXM} 20$ slLA. [Fecha revisión: 13 julio 2020].

VÉLEZ-RUIZ, R. I.; GONZALEZ, V. H.; ENGEL, M. S. 2013. Observations on the urban ecology of the Neotropical stingless bee Tetragonisca angustula (Hymenoptera: Apidae: Meliponini). Journal of Melittology 15: 1-8. https://doi.org/10.17161/jom. v0i15.4528.

WCISLO, W. T. 1996. Parasitism rates in relation to nest site in bees and wasps (Hymenoptera: Apoidea). Journal of Insect Behavior 9 (4): 643-656. https://doi.org/10.1007/BF02213885.

WINFREE, R.; BARTOMEUS, I.; CARIVEAU, D. P. 2011. Native pollinators in anthropogenic habitats. Annual Review of Ecology, Evolution, and Systematics 42 (1): 1-22. https://doi.org/10.1146/ annurev-ecolsys-102710-145042.

WRATTEN, S. D.; GILLESPIE, M.; DECOURTYE, A.; MADER, E.; DESNEUX, N. 2012. Pollinator habitat enhancement: Benefits to other ecosystem services. Agriculture, Ecosystems and Environment 159: 112-122. https://doi.org/10.1016/j. agee.2012.06.020.

YUCA-RIVAS, R. 2018. Pólen de las angiospermas del Valle Sagrado de los Incas vol. I y II. Fieldmuseum, fieldguides: 1023. Disponible en: https://www.researchgate.net/ publication/341614853_POLEN_de_las_angiospermas del_Valle_Sagrado_de_los_Incas_vol_II_Cusco_PERU [Fechā revisión: $\overline{1} 8$ septiembre $20 \overline{1}$ ]

\section{Origen y financiación}

Este trabajo fue financiado parcialmente con recursos del Proyecto de inversión 1121 - Investigación para la conservación de los ecosistemas y la flora de Bogotá D.C. y la región, 2016-2020, de la Subdirección Cientifica del Jardín Botánico de Bogotá José Celestino Mutis.

\section{Contribuciones de los autores}

Ángela Rodríguez: conceptualización, investigación, metodología, recursos, supervisión, visualización, escritura (borrador original), escritura (correcciones del arbitraje y de edición); Viviana García: curaduría de datos, investigación, metodología, visualización, escritura (correcciones del arbitraje y de edición); Susana Currea: curaduría de datos, análisis formal, investigación, visualización, escritura (borrador original), escritura (correcciones del arbitraje y de edición).

\section{Conflictos de interés}

Los autores declaramos no tener conflictos de intereses. 